\title{
High Order Mixed Finite Elements with Mass Lumping for Elasticity on Triangular Grids
}

\author{
Yan Yang ${ }^{1}$ and Xiaoping Xie ${ }^{2, *}$ \\ ${ }^{1}$ School of Sciences, Southwest Petroleum University, Chengdu 610500, China \\ ${ }^{2}$ School of Mathematics, Sichuan University, Chengdu 610064, China
}

Received 12 April 2021; Accepted (in revised version) 26 November 2021

\begin{abstract}
A family of conforming mixed finite elements with mass lumping on triangular grids are presented for linear elasticity. The stress field is approximated by symmetric $H(\operatorname{div})-P_{k}(k \geq 3)$ polynomial tensors enriched with higher order bubbles so as to allow mass lumping, and the displacement field is approximated by $C^{-1}-P_{k-1}$ polynomial vectors enriched with higher order terms. For both the proposed mixed elements and their mass lumping schemes, optimal error estimates are derived for the stress and displacement in $H$ (div) norm and $L^{2}$ norm, respectively. Numerical results confirm the theoretical analysis.
\end{abstract}

AMS subject classifications: 65N15, 65N30, 74H15, 74S05

Key words: Linear elasticity, mixed finite element, mass lumping, error estimate.

\section{Introduction}

Let $\Omega \subset \mathbb{R}^{2}$ be a polygonal region with boundary $\partial \Omega$. We consider the following mixed variational system of linear elasticity based on the Helligner-Reissner principle: Find $(\sigma, u) \in \Sigma \times V:=H(\operatorname{div}, \Omega ; \mathbb{S}) \times L^{2}\left(\Omega ; \mathbb{R}^{2}\right)$, such that

$$
\begin{cases}(\mathcal{A} \sigma, \tau)+(\operatorname{div} \tau, u)=0, & \forall \tau \in \Sigma, \\ -(\operatorname{div} \sigma, v)=(f, v), & \forall v \in V .\end{cases}
$$

Here $\sigma: \Omega \rightarrow \mathbb{S}:=\mathbb{R}_{\text {sym }}^{2 \times 2}$ denotes the symmetric $2 \times 2$ stress tensor field, $u: \Omega \rightarrow \mathbb{R}^{2}$ the displacement field, and $\mathcal{A} \sigma \in \mathbb{S}$ the compliance tensor with

$$
\mathcal{A} \sigma:=\frac{1}{2 \mu}\left(\sigma-\frac{\lambda}{2 \mu+2 \lambda} \operatorname{tr}(\sigma) I\right),
$$

where $\lambda>0, \mu>0$ are the Lamé coefficients, $\operatorname{tr}(\sigma)$ the trace of $\sigma, I$ the $2 \times 2$ iden-

\footnotetext{
${ }^{*}$ Corresponding author. Email addresses: yyan2011@163.com (Y. Yang), xpxie@scu.edu.cn (X. Xie) 
tity matrix, and $f$ the body force. $H(\operatorname{div}, \Omega ; \mathbb{S})$ denotes the space of square-integrable symmetric matrix fields with square-integrable divergence, and $L^{2}\left(\Omega ; \mathbb{R}^{2}\right)$ the space of square-integrable vector fields. The $L^{2}$ inner products on vector and matrix fields are given by

$$
\begin{array}{lll}
(v, w):=\int_{\Omega} v \cdot w d x=\int_{\Omega} \sum_{i=1}^{2} v_{i} w_{i} d x, & v=\left(v_{1}, v_{2}\right), & w=\left(w_{1}, w_{2}\right) \in V, \\
(\sigma, \tau):=\int_{\Omega} \sigma: \tau d x=\int_{\Omega} \sum_{1 \leq i, j \leq 2} \sigma_{i j} \tau_{i j} d x, & \sigma=\left(\sigma_{i j}\right), & \tau=\left(\tau_{i j}\right) \in \Sigma
\end{array}
$$

respectively.

According to the standard theory of mixed methods [11], a mixed finite element discretization of the weak problem (1.1) requires the pair of stress and displacement approximations to satisfy two stability conditions, i.e. a coercivity condition and an inf-sup condition. These stability constraints make it challengeable to construct stable finite element pairs with symmetric stresses. In this field, we refer to $[1-7,12,20-26,31$, 32] for some conforming or nonconforming mixed methods for elasticity. In particular, $\mathrm{Hu}$ and Zhang $[25,26]$ designed a family of conforming symmetric mixed finite elements with optimal convergence orders for linear elasticity on triangular and tetrahedral grids. Later $\mathrm{Hu}$ [21] extended the elements to simplicial grids in $\mathbb{R}^{n}$ for any positive integer $n$. In these elements, the stress is approximated by symmetric $H(\operatorname{div}, \Omega ; \mathbb{S})-P_{k}$ polynomial tensors and the displacement is approximated by $L^{2}\left(\Omega ; \mathbb{R}^{n}\right)-P_{k-1}$ polynomial vectors for $k \geq n+1$.

However, for a mixed finite element discretization based on (1.1), a computational drawback is the need to solve an algebraic system of saddle point type like

$$
\left(\begin{array}{cc}
\mathbb{A} & \mathbb{B}^{T} \\
-\mathbb{B} & \mathbb{O}
\end{array}\right)\left(\begin{array}{l}
X_{1} \\
X_{2}
\end{array}\right)=\left(\begin{array}{c}
O \\
F
\end{array}\right)
$$

where $\mathbb{A}$ is a symmetric and positive definite (SPD) matrix corresponding to the term $(\mathcal{A} \sigma, \tau)$ in (1.1), and $X_{1}$ and $X_{2}$ are the vectors of unknowns for the discrete stress and displacement approximations, respectively. One possible approach to resolve this difficulty is to apply "mass lumping" on $(\mathcal{A} \sigma, \tau)$ so as to get a diagonal or block-diagonal matrix approximation, $\tilde{\mathbb{A}}$, of the 'mass matrix' $\mathbb{A}$. Replacing $\mathbb{A}$ with $\tilde{\mathbb{A}}$ in the discrete system (1.3), we obtain

$$
X_{1}=-\tilde{\mathbb{A}}^{-1} \mathbb{B}^{T} X_{2}
$$

and then

$$
\mathbb{B} \tilde{\mathbb{A}}^{-1} \mathbb{B}^{T} X_{2}=F .
$$

Notice that $\tilde{\mathbb{A}}$ is diagonal or block-diagonal, so is $\tilde{\mathbb{A}}^{-1}$. This means that the Schur complement $\mathbb{B}^{-1} \mathbb{B}^{T}$ is SPD. As a result, by mass lumping the saddle point system (1.3) is reduced to the SPD system (1.4), which can be solved efficiently by many fast algorithms. 
The key to achieve mass lumping is to select appropriate numerical quadrature rule, in which the quadrature nodes are required to match the finite element basis functions as well as maintain sufficient numerical integration accuracy. It has been shown that mass lumping schemes can be constructed for some finite elements $[8,9$, 13-19, 27-29, 33]. In $[8,14,18]$ the standard linear triangular/tetrahedral elements with mass lumping were analyzed, where the quadrature nodes are the vertices of the elements. Traditional higher order elements are not suitable for mass lumping due to the requirements of numerical accuracy and stability, and one has to use finite element spaces enriched with some bubble functions to adapt mass lumping [13, 16, 19, 27$29]$. We note that a family of mixed rectangular and cubic finite elements with mass lumping were constructed in [9] for linear elastodynamic problems, where the stress and displacement are approximated by symmetric $H(\operatorname{div})-Q_{k}$ polynomial tensors and $L^{2}-Q_{k-1}$ polynomial vectors, respectively, and the locations of the degrees of freedom for the finite element spaces correspond to tensor products of one-dimensional quadrature nodes associated with Gauss-Lobatto (for stress) or Gauss-Legendre (for velocity) quadrature formulas.

In this paper, we first modify Hu-Zhang's mixed conforming finite elements [25] to obtain a family of new elements which allow mass lumping. The stress field is approximated by symmetric $H(\operatorname{div})-P_{k}(k \geq 3)$ polynomial tensors enriched with higher order bubbles, and the displacement field by $C^{-1}-P_{k-1}$ polynomial vectors enriched with higher order terms. Error analysis is carried out for the new elements as well as their mass lumping schemes.

The remainder of this paper is organized as follows. Section 2 introduces some preliminary results of mixed finite elements, including Hu-Zhang's elements. Sections 3 and 4 are devoted to the construction and analysis of the new mixed elements and their mass lumping schemes, respectively. Finally, Section 5 gives some numerical experiments to verify the theoretical results.

\section{Preliminaries}

\subsection{Notations}

For integer $m \geq 0$, let $H^{m}(\Omega ; X)$ be the Sobolev spaces consisting of functions with domain $\Omega$, taking values in $X=\mathbb{S}$ or $\mathbb{R}^{2}$, and with all derivatives of order at most $m$ square-integrable. The norm and semi-norm on $H^{m}(\Omega ; X)$ are denoted, respectively, by $\|\cdot\|_{m}$ and $|\cdot|_{m}$. In particular, $H^{0}(\Omega ; X)=L^{2}(\Omega ; X)$.

Suppose $\mathcal{T}_{h}=\cup\{K\}$ to be a conforming and shape-regular triangulation of the domain $\Omega$ consisting of triangles. For any $K \in \mathcal{T}_{h}$, let $h_{K}$ denote its diameter, and set $h:=\max _{K \in \mathcal{T}_{h}} h_{K}$. We use $P_{m}(K ; X)$ to denote the set of all polynomials on $K$ with degree at most $m$ and taking values in $X$. Throughout the paper, we use $a \lesssim b(a \gtrsim b)$ to denote $a \leq C b(a \geq C b)$, where $C$ is a generic positive constant independent of mesh parameters $h$. 


\subsection{Mixed finite element discretization}

Let $\Sigma_{h} \subset \Sigma, V_{h} \subset V$ be two finite-dimensional spaces for the stress and displacement approximations, respectively. Then the mixed finite element discretization of (1.1) reads: Find $\left(\sigma_{h}, u_{h}\right) \in \Sigma_{h} \times V_{h}$ such that

$$
\begin{cases}\left(\mathcal{A} \sigma_{h}, \tau_{h}\right)+\left(\operatorname{div} \tau_{h}, u_{h}\right)=0, & \forall \tau_{h} \in \Sigma_{h}, \\ -\left(\operatorname{div} \sigma_{h}, v_{h}\right)=\left(f, v_{h}\right), & \forall v_{h} \in V_{h} .\end{cases}
$$

According to the standard theory of mixed finite element methods $[10,11]$, the pair of finite element spaces $\Sigma_{h}$ and $V_{h}$ needs to satisfy the following stability conditions:

- K-ellipticity condition

$$
\left(\mathcal{A} \tau_{h}, \tau_{h}\right) \gtrsim\left\|\tau_{h}\right\|_{H(\operatorname{div})}^{2}, \quad \forall \tau_{h} \in Z_{h}:=\left\{\tau_{h} \in \Sigma_{h} \mid\left(\operatorname{div} \tau_{h}, v\right)=0, \forall v \in V_{h}\right\},
$$

where $\|\cdot\|_{H(\text { div })}$ is the norm on the space $\Sigma$ defined by

$$
\|\tau\|_{H(\operatorname{div})}^{2}:=\|\tau\|_{0}^{2}+\|\operatorname{div} \tau\|_{0}^{2}, \quad \forall \tau \in \Sigma .
$$

- Discrete BB ( inf-sup ) condition

$$
\sup _{\tau_{h} \in \Sigma_{h}} \frac{\left(\operatorname{div} \tau_{h}, v_{h}\right)}{\left\|\tau_{h}\right\|_{H(\operatorname{div})}} \gtrsim\left\|v_{h}\right\|_{0}, \quad \forall v_{h} \in V_{h} .
$$

\subsection{Hu-Zhang's mixed conforming elements}

For each $K \in \mathcal{T}_{h}$, define an $H\left(\right.$ div) bubble function space, $B_{k, K}$, of polynomials of degree $k$ by

$$
B_{k, K}:=\left\{\tau \in P_{k}(K ; \mathbb{S}):\left.\tau \nu\right|_{\partial K}=0\right\},
$$

where $\nu$ is the normal vector along $\partial K$. Introduce the local rigid motion space

$$
\begin{aligned}
R(K) & :=\left\{v \in H^{1}\left(K ; \mathbb{R}^{2}\right): \nabla v+(\nabla v)^{T}=0\right\} \\
& =\operatorname{span}\left\{\left(\begin{array}{l}
1 \\
0
\end{array}\right),\left(\begin{array}{l}
0 \\
1
\end{array}\right),\left(\begin{array}{r}
-x_{2} \\
x_{1}
\end{array}\right)\right\}
\end{aligned}
$$

and its orthogonal complement space with respect to $P_{k-1}\left(K ; \mathbb{R}^{2}\right)(k \geq 2)$,

$$
R_{k}^{\perp}(K)=\left\{v \in P_{k-1}\left(K ; \mathbb{R}^{2}\right):(v, w)_{K}=0, \forall w \in R(K)\right\} .
$$

The following result holds.

Lemma 2.1 ([21]). For any $K \in \mathcal{T}_{h}$ and $k \geq 2$, it holds that

$$
R_{k}^{\perp}(K)=\operatorname{div} B_{k, K} .
$$


For $k \geq 3$, introduce the following global finite element spaces [25]:

$$
\begin{aligned}
\Sigma_{k, h} & :=\widetilde{\Sigma}_{k, h}+B_{k, h}, \\
V_{k, h} & :=\left\{v \in L^{2}\left(\Omega ; \mathbb{R}^{2}\right):\left.v\right|_{K} \in P_{k-1}\left(K ; \mathbb{R}^{2}\right), \forall K \in \mathcal{T}_{h}\right\},
\end{aligned}
$$

where

$$
\begin{aligned}
& B_{k, h}:=\left\{\tau \in H(\operatorname{div}, \Omega ; \mathbb{S}):\left.\tau\right|_{K} \in B_{k, K}, \forall K \in \mathcal{T}_{h}\right\}, \\
& \widetilde{\Sigma}_{k, h}:=\left\{\tau \in H^{1}(\Omega ; \mathbb{S}):\left.\tau\right|_{K} \in P_{k}(K ; \mathbb{S}), \forall K \in \mathcal{T}_{h}\right\} .
\end{aligned}
$$

It is easy to see that $\mathbb{S}$ has a canonical basis

$$
\mathbb{T}_{1}:=\left(\begin{array}{cc}
1 & 0 \\
0 & 0
\end{array}\right), \quad \mathbb{T}_{2}:=\left(\begin{array}{cc}
0 & 1 \\
1 & 0
\end{array}\right), \quad \mathbb{T}_{3}:=\left(\begin{array}{cc}
0 & 0 \\
0 & 1
\end{array}\right) .
$$

For any edge $E$ of $\mathcal{T}_{h}$, let $t_{E}$ and $\nu_{E}$ be its unit tangent and norm vectors, respectively. Define

$$
T_{E}:=t_{E} t_{E}^{T}
$$

Let $T_{E, j}^{\perp} \in \mathbb{S}(j=1,2)$ be two orthogonal complement matrices of $T_{E}$ with

$$
T_{E, j}^{\perp}: T_{E}=0, \quad T_{E, j}^{\perp}: T_{E, j}^{\perp}=1, \quad T_{E, 1}^{\perp}: T_{E, 2}^{\perp}=0 .
$$

Here $A: B=\sum_{i=1}^{n} \sum_{i=1}^{n} a_{i j} b_{i j}$ for $A=\left(a_{i j}\right)_{i, j=1}^{n}$ and $B=\left(b_{i j}\right)_{i, j=1}^{n}$. It has been shown in $[25]$ that

$$
\mathbb{S}=\operatorname{span}\left\{\mathbb{T}_{1}, \mathbb{T}_{2}, \mathbb{T}_{3}\right\}=\operatorname{span}\left\{T_{E}, T_{E, 1}^{\perp}, T_{E, 2}^{\perp}\right\}
$$

Let $\left\{\chi_{i}\right\}_{i=1}^{l}$ be the set of nodes for the Lagrange element of order $k$ and $\left\{\zeta_{i}\right\}_{i=1}^{l}$ be their associated Lagrange node basis functions such that

$$
\zeta_{i}\left(\chi_{j}\right)=\delta_{i j}, \quad i, j=1, \ldots, l .
$$

Then the basis functions of $\Sigma_{k, h}$ on $K$ fall into the following four classes [25]:

(1) Vertex-based basis functions. If $\chi_{i}$ is a vertex, the three associated basis functions of $\Sigma_{k, h}$ are $\zeta_{i} \mathbb{T}_{j}, j=1,2,3$.

(2) Volumed-based functions. If $\chi_{i}$ is a node inside $K$, the three associated basis functions of $\Sigma_{k, h}$ are $\zeta_{i} \mathbb{T}_{j}, j=1,2,3$.

(3) Edge-based basis functions with nonzero fluxes. If $\chi_{i}$ is a node on edge $E$ (not the vertex), the two associated basis functions of $\Sigma_{k, h}$ are $\zeta_{i} T_{E, j}^{\perp}, j=1,2$.

(4) Edge-based bubble functions. If $\chi_{i} \in K$ is a node on edge $E$ (not the vertex) shared by elements $K_{1}$ and $K_{2}$, then $\left.\zeta_{i} T_{E} \nu_{E}\right|_{E} \equiv 0$ due to (2.11), and then the $H$ (div) bubble functions in $\Sigma_{k, h}$ are $\varkappa_{j} \zeta_{i} T_{E}, j=1,2$, where $\varkappa_{j}$ is the characteristic function of $K_{j}$. 
Theorem 2.1 ([21,25]). Let $(\sigma, u) \in \Sigma \times V$ and $\left(\sigma_{h}, u_{h}\right) \in \Sigma_{h} \times V_{h}$, with

$$
\Sigma_{h}=\Sigma_{k, h}, \quad V_{h}=V_{k, h}
$$

solve (1.1) and (2.1), respectively. If $\sigma \in H^{k+1}(\Omega ; \mathbb{S})$ and $v \in H^{k}\left(\Omega ; \mathbb{R}^{2}\right)$, then

$$
\begin{aligned}
& \left\|\sigma-\sigma_{h}\right\|_{H(\mathrm{div})}+\left\|u-u_{h}\right\|_{0} \lesssim h^{k}\left(\|\sigma\|_{k+1}+\|u\|_{k}\right), \\
& \left\|\sigma-\sigma_{h}\right\|_{0} \lesssim h^{k+1}\|\sigma\|_{k+1} .
\end{aligned}
$$

\subsection{Mass lumping for Hu-Zhang elements?}

To solve the discrete system (2.1), we need to compute the inverse of the mass matrix corresponding to the term $\left(\mathcal{A} \sigma_{h}, \tau_{h}\right)$. Let us first consider the local mass matrix on element $K \in \mathcal{T}_{h}$. Recall that $\chi_{i}(i=1, \ldots, l)$ are the nodes of the Lagrange element of order $k$ and $\zeta_{i}(i=1, \ldots, l)$ are the associated Lagrange node basis functions. Thus, the basis functions of $\Sigma_{k, h}$ on $K$ can be denoted by

$$
\varphi_{3(m-1)+s}:=\zeta_{m} T_{s}, \quad m=1, \ldots, l, \quad s=1,2,3,
$$

where $T_{s} \in\left\{\mathbb{T}_{1}, \mathbb{T}_{2}, \mathbb{T}_{3}\right\}$ if $\chi_{m}$ is a vertex or a node inside $K$, and $T_{s} \in\left\{T_{E}, T_{E, 1}^{\perp}, T_{E, 2}^{\perp}\right\}$ if $\chi_{m}$ is a node on edge $E$ (not the vertex). Then the local mass matrix $A_{K}$ on $K$ is given by

$$
\left(A_{K}\right)_{i j}:=\left.\left(\mathcal{A} \varphi_{i}, \varphi_{j}\right)\right|_{K}, \quad i, j=1, \ldots, 3 l .
$$

During the finite element method, we commonly evaluate the integrals approximately by using a numerical integration formula in each element $K$. To achieve mass lumping, the usual way is to choose the quadrature points to be the nodes $\left\{\chi_{i}\right\}_{i=1}^{l}$ on $K$, and the quadrature rule is of the form

$$
\int_{K} f d x \approx I_{k, K}(f):=\sum_{i=1}^{l} w_{i} f\left(\chi_{i}\right),
$$

where $\left\{w_{i}\right\}_{i=1}^{l}$ are the weights. Then we have

$$
I_{k, K}\left(\zeta_{i}, \zeta_{j}\right)=w_{i} \delta_{i j}, \quad i, j=1, \ldots, l .
$$

For $m, n=1, \ldots, l$ and $s, q=1,2,3$, set

$$
i=3(m-1)+s, \quad j=3(n-1)+q,
$$

then from (2.17) it follows:

$$
\begin{aligned}
\left(A_{K}\right)_{i j} \approx\left(\tilde{A}_{K}\right)_{i j}: & =I_{k, K}\left(\mathcal{A} \varphi_{i}, \varphi_{j}\right)=I_{k, K}\left(\mathcal{A} \zeta_{m} T_{s}, \zeta_{n} T_{q}\right) \\
& = \begin{cases}0, & m \neq n \\
w_{m}\left(\mathcal{A} T_{s}: T_{q}\right), & m=n\end{cases}
\end{aligned}
$$


This means that the approximate local mass matrix $\tilde{A}_{k}$ is block-diagonal and of the form

$$
\tilde{A}_{K}=\operatorname{diag}\left(w_{1} B_{1}, w_{2} B_{2}, \cdots, w_{l} B_{l}\right),
$$

where $B_{m}(m=1, \ldots, l)$ are $3 \times 3$ SPD matrices. For example, if $\chi_{m}$ is a vertex or a node inside $K$, then

$$
B_{m}=\frac{1}{4 \mu(\mu+\lambda)}\left(\begin{array}{ccc}
2 \mu+\lambda & 0 & -\lambda \\
0 & 4(\mu+\lambda) & 0 \\
-\lambda & 0 & 2 \mu+\lambda
\end{array}\right) .
$$

However, the accuracy of numerical integration has to be taken into account. From the standard theory $[8,14,30]$, the following condition is required to satisfy so as to maintain the accuracy of the scheme (2.1):

(A1) The quadrature rule (2.16) must be exact for $P_{2 k-2}$.

Unfortunately, the standard $P_{k}$ Lagrange elements fail to satisfy this condition for $k \geq 3$ (cf. [16]). In other words, Hu-Zhang's elements do not allow mass lumping without loss of numerical accuracy.

\section{Modified mixed conforming finite elements for elasticity}

\section{1. $P_{k, k^{\prime}}$-Lagrange finite elements for mass lumping}

As mentioned before, the standard $P_{k}$ Lagrange elements with $k \geq 3$ fail to satisfy the accuracy condition, (A1), of the quadrature rule (2.16) for mass lumping. For wave problems, as shown in $[13,16,19,27-29]$, an efficient way to address this difficulty is to construct a slightly larger finite element space

$$
P_{k, k^{\prime}}(K ; \mathbb{R}):=P_{k}(K ; \mathbb{R})+b P_{k^{\prime}-3}(K ; \mathbb{R})=P_{k}(K ; \mathbb{R}) \oplus b \sum_{i=k-2}^{k^{\prime}-3} P_{i}^{h o m}(K ; \mathbb{R}) .
$$

Here $k^{\prime}>k$, and $b=\lambda_{1} \lambda_{2} \lambda_{3}$ is the bubble function on the element $K$ with $\lambda_{i}(i=$ $1,2,3)$ being the barycentric coordinates. $P_{i}^{\text {hom }}(K ; \mathbb{R})$ denotes the set of homogeneous polynomials on $K$ of degree $i$. The symbol " $\oplus$ " means that $P_{k}(K ; \mathbb{R}) \cap b P_{i}^{h o m}(K ; \mathbb{R})=$ $\{0\}$ for $i=k-2, k-1, \ldots, k^{\prime}-3$.

Let $\left\{\chi_{i}\right\}_{i=1}^{r}$ be the set of nodes for the $P_{k, k^{\prime}}$-Lagrange element. Then the corresponding quadrature rule is of the form

$$
\int_{K} f d x \approx I_{k, k^{\prime}, K}(f):=\sum_{i=1}^{r} w_{i} f\left(\chi_{i}\right)
$$

where $\left\{w_{i}\right\}_{i=1}^{r}$ are the weights, and $\sum_{i=1}^{r} w_{i}=\operatorname{meas}(K)$. To maintain the accuracy and stability of finite element scheme, the following two conditions are required (cf. $[13,16])$ : 
(B1) The weights $w_{i}(i=1, \ldots, r)$ in (3.2) should be strictly positive.

(B2) The quadrature rule (3.2) must be exact for $P_{k+k^{\prime}-2}$.

Table 1 lists several $P_{k, k^{\prime}}$ finite elements which satisfy (B1) and (B2) with $3 \leq k \leq 5$. In the table, a given node $\left(\alpha_{1}, \alpha_{2}, \alpha_{3}\right)$ represents an equivalence class which includes all the nodes obtained by taking all the permutations of the barycentric coordinates $\alpha_{i}$. For instance, the class $(0,0,1)$ includes three points, $(0,0,1),(0,1,0)$, and $(1,0,0)$; the class $(\alpha, 0,1-\alpha)$ includes

$$
\begin{array}{lll}
(\alpha, 0,1-\alpha), & (0, \alpha, 1-\alpha), & (\alpha, 1-\alpha, 0), \\
(1-\alpha, \alpha, 0), & (1-\alpha, 0, \alpha), & (0,1-\alpha, \alpha) .
\end{array}
$$

Fig. 1 shows the distributions of nodes for the elements of $P_{3,4}, P_{3,5}$ and $P_{4,5}$.

\begin{tabular}{|c|c|c|c|c|}
\hline space & $k k^{\prime}$ & class & weight & position parameters \\
\hline \multirow[t]{3}{*}{$P_{3,4}[13,16]$} & \multirow[t]{3}{*}{34} & $(0,0,1)$ & $(8-\sqrt{7}) / 720$ & \multirow{3}{*}{$\begin{array}{c}1 / 2-\sqrt{1 /(3 \sqrt{7})-1 / 12} \\
(7-\sqrt{7}) / 21\end{array}$} \\
\hline & & $(\alpha, 0,1-\alpha)$ & $(7+4 \sqrt{7}) / 720$ & \\
\hline & & $(\alpha, \alpha, 1-2 \alpha)$ & $7(14-\sqrt{7}) / 720$ & \\
\hline \multirow[t]{4}{*}{$P_{3,5}[13]$} & \multirow[t]{4}{*}{35} & $(0,0,1)$ & 0.00356517965360224101681201 & \multirow{4}{*}{$\begin{array}{l}0.307745941625991646104616 \\
0.118613686396592868190663 \\
0.425340125989747152025431\end{array}$} \\
\hline & & $(\alpha, 0,1-\alpha)$ & 0.0147847080884026469663777 & \\
\hline & & $(\alpha, \alpha, 1-2 \alpha)$ & 0.0509423265134759070757019 & \\
\hline & & $(\alpha, \alpha, 1-2 \alpha)$ & 0.0825897443227832246413973 & \\
\hline \multirow[t]{5}{*}{$\overline{P_{4,5}[13,29]}$} & \multirow[t]{5}{*}{45} & $(0,0,1)$ & $1 / 315$ & \multirow{5}{*}{$\begin{array}{l}1 / 2(1-1 / \sqrt{3}) \\
(5-\sqrt{7}) / 18 \\
(5+\sqrt{7}) / 18\end{array}$} \\
\hline & & $(1 / 2,0,1 / 2)$ & $4 / 315$ & \\
\hline & & $(\alpha, 0,1-\alpha)$ & $3 / 280$ & \\
\hline & & $(\alpha, \alpha, 1-2 \alpha)$ & $163 / 2520-47 \sqrt{7} / 8820$ & \\
\hline & & $(\alpha, \alpha, 1-2 \alpha)$ & $163 / 2520+47 \sqrt{7} / 8820$ & \\
\hline \multirow[t]{6}{*}{$P_{4,6}[29]$} & \multirow[t]{6}{*}{46} & $(0,0,1)$ & 0.00150915593385883937469324 & \multirow{6}{*}{\begin{tabular}{|c}
0.199632107119457219140683 \\
0.0804959191700374444460458 \\
0.107591821784867520262175 \\
0.302912783038363411733216
\end{tabular}} \\
\hline & & $(1 / 2,0,1 / 2)$ & 0.0101871481261788846308014 & \\
\hline & & $(\alpha, 0,1-\alpha)$ & 0.00699540146387514358396201 & \\
\hline & & $(1 / 3,1 / 3,1 / 3)$ & 0.0660095591593093891810431 & \\
\hline & & $(\alpha, \alpha, 1-2 \alpha)$ & 0.0234436060814549086935898 & \\
\hline & & $(\alpha, \beta, 1-\alpha-\beta)$ & 0.0477663836054936418696553 & \\
\hline \multirow[t]{7}{*}{$\overline{P_{5,7}[13,29]}$} & \multirow[t]{7}{*}{57} & $(0,0,1)$ & 0.000709423970679245979296007 & \\
\hline & & $(\alpha, 0,1-\alpha)$ & 0.00348057864048921065844268 & 0.132264581632713985353888 \\
\hline & & $(\alpha, 0,1-\alpha)$ & 0.00619056500367662911411813 & 0.363298074153686045705506 \\
\hline & & $(\alpha, \alpha, 1-2 \alpha)$ & 0.0116261354596175711394984 & 0.0575276844114101056608175 \\
\hline & & $(\alpha, \alpha, 1-2 \alpha)$ & 0.0459012376307628573770191 & 0.256859107261959076063891 \\
\hline & & $(\alpha, \alpha, 1-2 \alpha)$ & 0.0345304303772827935283885 & 0.457836838079161101938503 \\
\hline & & $(\alpha, \beta, 1-\alpha-\beta)$ & 0.0272785759699962595486715 & $\begin{array}{c}0.0781925836255170219988860 \\
0.221001218759890007978128\end{array}$ \\
\hline
\end{tabular}

Table 1: $P_{k, k^{\prime}}$-Lagrange triangular elements.

\subsection{Modified mixed element spaces for elasticity}

Inspired by the $P_{k, k^{\prime}}$-Lagrange elements which allow mass lumping, in this subsection we shall construct a family of new mixed conforming element spaces based on the modification of Hu-Zhang's elements. 

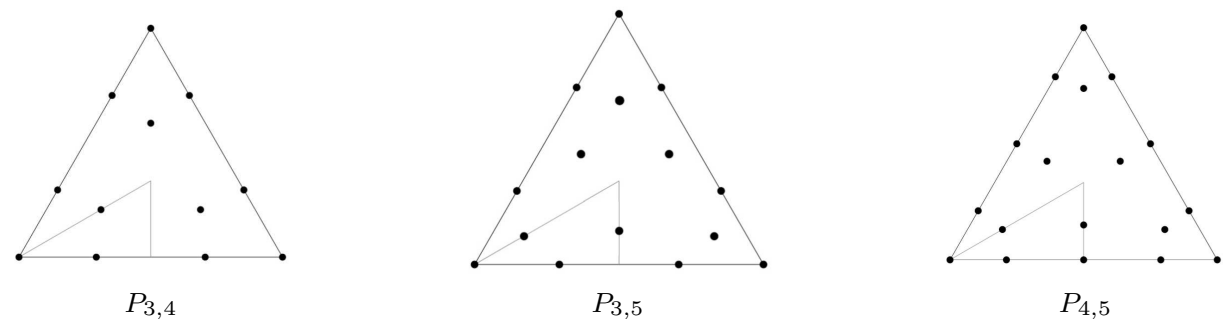

Figure 1: Nodes for the elements, mapped to the equilateral triangle.

For $k^{\prime}>k \geq 3$, set

$$
\Lambda_{k, k^{\prime}}:=\left\{\tau \in H(\operatorname{div}, \Omega ; \mathbb{S}):\left.\tau\right|_{K}=\sum_{i=k-2}^{k^{\prime}-3} b P_{i}^{h o m}(K ; \mathbb{S}), \forall K \in \mathcal{T}_{h}\right\} .
$$

Then the modified global finite element spaces for the stress and displacement are given by

$$
\begin{aligned}
\Sigma_{k, k^{\prime}, h} & :=\Sigma_{k, h} \oplus \Lambda_{k, k^{\prime}}, \\
V_{k, k^{\prime}, h} & :=V_{k, h}+\operatorname{div} \Lambda_{k, k^{\prime}} .
\end{aligned}
$$

Obviously we have $\operatorname{div} \Sigma_{k, k^{\prime}, h} \subset V_{k, k^{\prime}, h}$.

Remark 3.1. If we define

$$
\begin{aligned}
& B_{k, k^{\prime}, h}:=\left\{\tau \in P_{k, k^{\prime}}(K ; \mathbb{S}):\left.\tau \nu\right|_{\partial K}=0, \forall K \in \mathcal{T}_{h}\right\}=B_{k, h}+\Lambda_{k, k^{\prime}}, \\
& \widetilde{\Sigma}_{k, k^{\prime}, h}:=\left\{\tau \in H^{1}(\Omega ; \mathbb{S}):\left.\tau\right|_{K} \in P_{k, k^{\prime}}(K ; \mathbb{S}), \forall K \in \mathcal{T}_{h}\right\}=\widetilde{\Sigma}_{k, h}+\Lambda_{k, k^{\prime}},
\end{aligned}
$$

then we can also write

$$
\Sigma_{k, k^{\prime}, h}=\widetilde{\Sigma}_{k, k^{\prime}, h}+B_{k, k^{\prime}, h} .
$$

Let $\left\{\chi_{i}\right\}$ be the set of the nodes for the $P_{k, k^{\prime}}$-Lagrange element, and $\left\{\zeta_{i}\right\}$ be the corresponding nodal basis functions satisfying

$$
\zeta_{i}\left(\chi_{j}\right)=\delta_{i j}
$$

Similarly to Hu-Zhang's elements described in Section 2.3, for each node $\chi_{i}$ the associated basis functions of $\Sigma_{k, k^{\prime}, h}$ on $K$ are given as follows:

(1) $\zeta_{i} \mathbb{T}_{j}(j=1,2,3)$, if $\chi_{i}$ is a vertex or a node inside $K$;

(2) $\zeta_{i} T_{E, j}^{\perp}(j=1,2)$ and $\varkappa_{j} \zeta_{i} T_{E}(j=1,2)$, if $\chi_{i}$ is a node on edge $E$ (not the vertex) shared by elements $K_{1}$ and $K_{2}$. Here we recall that $\varkappa_{j}$ is the characteristic function of $K_{j}$.

In Table 2, we give the numbers of degrees of freedom on each element $K$ and on $\mathcal{T}_{h}$ for several choices of finite element spaces, where $\mathbb{N}, \mathbb{E}$ and $\mathbb{K}$ denote respectively the numbers of nodes, edges and elements of $\mathcal{T}_{h}$. 
Table 2: Numbers of degrees of freedom on element $K$ and on $\mathcal{T}_{h}$.

\begin{tabular}{||l|c|c|c|c||}
\hline \multirow{2}{*}{} & \multicolumn{2}{|c|}{$K$} & \multicolumn{2}{c||}{$\mathcal{T}_{h}$} \\
\cline { 2 - 5 } & $\sigma_{h}$ & $u_{h}$ & $\sigma_{h}$ & $u_{h}$ \\
\hline$\Sigma_{3, h}-V_{3, h}$ & 30 & 12 & $9 \mathbb{N}+4 \mathbb{E}+9 \mathbb{K}$ & $12 \mathbb{K}$ \\
$\Sigma_{3,4, h}-V_{3,4, h}$ & 36 & 18 & $9 \mathbb{N}+4 \mathbb{E}+15 \mathbb{K}$ & $18 \mathbb{K}$ \\
$\Sigma_{3,5, h}-V_{3,5, h}$ & 45 & 27 & $9 \mathbb{N}+4 \mathbb{E}+24 \mathbb{K}$ & $27 \mathbb{K}$ \\
\hline$\Sigma_{4, h}-V_{4, h}$ & 45 & 20 & $9 \mathbb{N}+6 \mathbb{E}+18 \mathbb{K}$ & $20 \mathbb{K}$ \\
$\Sigma_{4,5, h}-V_{4,5, h}$ & 54 & 29 & $9 \mathbb{N}+6 \mathbb{E}+27 \mathbb{K}$ & $29 \mathbb{K}$ \\
\hline
\end{tabular}

\subsection{Stability results}

This subsection is devoted to the stability analysis and error estimation of the mixed finite element scheme (2.1) with

$$
\Sigma_{h}=\Sigma_{k, k^{\prime}, h}, \quad V_{h}=V_{k, k^{\prime}, h} .
$$

Let $\hat{K}$ be the reference element with vertexes $(0,0),(0,1),(1,0)$. For each $K \in \mathcal{T}_{h}$, let $F_{K}$ denote the affine map from $\hat{K}$ onto $K$ so that $F_{K}(\hat{K})=K$. Let $\chi_{0}, \chi_{1}, \chi_{2}$ be the vertices of triangle $K \in \mathcal{T}_{h}$. The referencing mapping is then of the form

$$
x=F_{K}(\hat{x})=\chi_{0}+\left(\begin{array}{cc}
\chi_{1}-\chi_{0} & \chi_{2}-\chi_{0}
\end{array}\right) \hat{x}:=\chi_{0}+B_{K} \hat{x}, \quad \forall \hat{x} \in \hat{K} .
$$

By the shape regularity of $\mathcal{T}_{h}$, it holds that

$$
\left\|B_{K}\right\|_{0} \lesssim h, \quad\left\|B_{K}^{-1}\right\|_{0} \lesssim h^{-1} .
$$

We need to introduce the Piola transform as follows. Given $\hat{\tau}: \hat{K} \mapsto \mathbb{S}, \tau: K \mapsto \mathbb{S}$ is defined by

$$
\tau(x):=B_{K} \hat{\tau}(\hat{x}) B_{K}^{T} .
$$

Clearly this sets up a one-to-one correspondence between $L^{2}(\hat{K} ; \mathbb{S})$ and $L^{2}(K ; \mathbb{S})$ with

$$
\operatorname{div} \tau(x)=B_{K} \widehat{\operatorname{div}} \hat{\tau}(\hat{x}) .
$$

Standard scaling arguments yield the following lemma.

Lemma 3.1. For any $K \in \mathcal{T}_{h}$ and $\hat{\tau} \in \hat{P}_{k, k^{\prime}}(\hat{K} ; \mathbb{S})$, let $\tau$ be given by (3.12). Then for $1 \leq q \leq k$,

$$
\begin{aligned}
&|\tau|_{q, K} \lesssim h^{2-q}\left|\operatorname{det} B_{K}\right|^{\frac{1}{2}}|\hat{\tau}|_{q, \hat{K}}, \\
&|\hat{\tau}|_{q, \hat{K}} \lesssim h^{q-2}\left|\operatorname{det} B_{K}\right|^{-\frac{1}{2}}|\tau|_{q, K} .
\end{aligned}
$$

Define the piecewise $m$-order semi-norm $|\cdot|_{m, h}(0 \leq m \leq k)$ on $\Sigma_{k, k^{\prime}, h}$ as follows:

$$
\left|\tau_{h}\right|_{m, h}:=\left(\sum_{K \in \mathcal{T}_{h}}\left|\tau_{h}\right|_{m, K}^{2}\right)^{\frac{1}{2}}, \quad \tau_{h} \in \Sigma_{k, k^{\prime}, h} .
$$


Lemma 3.2. For $\tau \in \Sigma_{k, k^{\prime}, h}$ satisfying

$$
\left.\operatorname{div} \tau\right|_{K}=0, \quad \forall K \in \mathcal{T}_{h} \Rightarrow \tau=0,
$$

it holds

$$
\|\tau\|_{0}+h|\tau|_{1, h} \lesssim h\|\operatorname{div} \tau\|_{0} .
$$

Proof. For any $K \in \mathcal{T}_{h}$, let $\hat{K}$ be the reference element. Then for $\tau \in \Sigma_{k, k^{\prime}, h}$ satisfying (3.17), $\|\widehat{\operatorname{div}} \hat{\tau}\|_{0, \hat{K}},|\hat{\tau}|_{1, \hat{K}}$ and $\|\hat{\tau}\|_{0, \hat{K}}$ are norms on $\hat{K}$, which means

$$
|\hat{\tau}|_{1, \hat{K}} \lesssim\|\hat{\tau}\|_{0, \hat{K}} \lesssim\|\widehat{\operatorname{div}} \hat{\tau}\|_{0, \hat{K}}
$$

On the other hand, by (3.12) and (3.13) we easily have

$$
\|\tau\|_{0, K} \lesssim h\|\hat{\tau}\|_{0, \hat{K}}, \quad|\tau|_{1, K} \lesssim|\hat{\tau}|_{1, \hat{K}}, \quad\|\widehat{\operatorname{div}} \hat{\tau}\|_{0, \hat{K}} \lesssim\|\operatorname{div} \tau\|_{0, K} .
$$

As a result,

$$
\|\tau\|_{0, K}+h|\tau|_{1, K} \lesssim h\|\operatorname{div} \tau\|_{0, K}
$$

This leads to the inequality (3.18).

In view of the definitions in (3.7) and (2.4), integration by part yields

$$
\int_{K} \operatorname{div} \tau_{h} \cdot w_{h} d x=0, \quad \forall \tau_{h} \in B_{k, k^{\prime}, h}, \quad w_{h} \in R(K), \quad K \in \mathcal{T}_{h} .
$$

Analogous to (2.5), we define

$$
R_{k, k^{\prime}}^{\perp}(K):=\left\{v \in V_{k, k^{\prime}, h}:(v, w)_{K}=0, \forall w \in R(K)\right\} .
$$

By following the same routines as in [21], we can easily derive the following two lemmas.

Lemma 3.3. For any $K \in \mathcal{T}_{h}$ and $k \geq 2$, it holds that

$$
R_{k, k^{\prime}}^{\perp}(K)=\left.\operatorname{div} B_{k, k^{\prime}, h}\right|_{K} .
$$

Lemma 3.4. For any $v_{h} \in V_{k, k^{\prime}, h}$, there exists $\tau_{h} \in \Sigma_{k, k^{\prime}, h}$ such that

$$
\int_{K}\left(\operatorname{div} \tau_{h}-v_{h}\right) \cdot w d x=0, \quad \forall w \in R(K), \quad K \in \mathcal{T}_{h}
$$

and

$$
\left\|\tau_{h}\right\|_{H(\text { div })} \lesssim\left\|v_{h}\right\|_{0}
$$

By using Lemmas 3.2-3.4 and following a similar way in [21], we can get the following existence and uniqueness result.

Theorem 3.1. The mixed finite element scheme (2.1) with $\Sigma_{h}=\Sigma_{k, k^{\prime}, h}$ and $V_{h}=V_{k, k^{\prime}, h}$ admits a unique solution $\left(\sigma_{h}, u_{h}\right) \in \Sigma_{h} \times V_{h}$. 
Remark 3.2. From Lemmas 3.3 and 3.4, we can derive that there exists an interpolation $\Pi_{h}: H^{1}(\Omega ; \mathbb{S}) \mapsto \Sigma_{k, k^{\prime}, h}$ such that for any $\tau \in H^{1}(\Omega ; \mathbb{S})$,

$$
\left(\operatorname{div}\left(\tau-\Pi_{h} \tau\right), v_{h}\right)_{K}=0, \quad \forall K \in \mathcal{T}_{h}, \quad \forall v_{h} \in V_{k, k^{\prime}, h}
$$

Furthermore, if $\tau \in H^{k+1}(\Omega ; \mathbb{S})$, then

$$
\left\|\tau-\Pi_{h} \tau\right\|_{0} \leq C h^{k+1}\|\tau\|_{k+1} .
$$

This shows that the operator $\Pi_{h}: H^{1}(\Omega ; \mathbb{S}) \mapsto \Sigma_{k, k^{\prime}, h}$ has the following commutative property:

$$
P_{h} \operatorname{div} \tau=\operatorname{div} \Pi_{h} \tau, \quad \forall \tau \in H^{1}(\Omega ; \mathbb{S}) .
$$

Here $P_{h}: L^{2}\left(\Omega ; \mathbb{R}^{2}\right) \mapsto V_{k, k^{\prime}, h}$ is the $L^{2}$ projection operator.

By the stability conditions (2.2)-(2.3) and Remark 3.2, we can easily obtain the following error estimates.

Theorem 3.2. Let $(\sigma, u) \in\left(\Sigma \cap H^{k+1}(\Omega ; \mathbb{S})\right) \times\left(V \cap H^{k}\left(\Omega ; \mathbb{R}^{2}\right)\right)$ and $\left(\sigma_{h}, u_{h}\right) \in \Sigma_{h} \times V_{h}=$ $\Sigma_{k, k^{\prime}, h} \times V_{k, k^{\prime}, h}$ solve (1.1) and (2.1), respectively. Then

$$
\begin{aligned}
& \left\|\sigma-\sigma_{h}\right\|_{H(\mathrm{div})}+\left\|u-u_{h}\right\|_{0} \lesssim h^{k}\left(\|\sigma\|_{k+1}+\|u\|_{k}\right), \\
& \left\|\sigma-\sigma_{h}\right\|_{0} \lesssim h^{k+1}\|\sigma\|_{k+1} .
\end{aligned}
$$

\section{Mass lumping mixed finite element method}

\subsection{Mass lumping scheme}

As mentioned before, the mixed scheme (2.1) leads to an algebraic system of saddle point type. One approach to address this issue is applying mass lumping.

The mass lumping scheme for (2.1) is described as follows: Find $\left(\sigma_{h}, u_{h}\right) \in \Sigma_{k, k^{\prime}, h} \times$ $V_{k, k^{\prime}, h}$, such that

$$
\begin{cases}\left(\mathcal{A} \sigma_{h}, \tau_{h}\right)_{h}+\left(\operatorname{div} \tau, u_{h}\right)=0, & \forall \tau_{h} \in \Sigma_{k, k^{\prime}, h}, \\ -\left(\operatorname{div} \sigma_{h}, v_{h}\right)=\left(f, v_{h}\right), & \forall v_{h} \in V_{k, k^{\prime}, h} .\end{cases}
$$

Here $\left(\mathcal{A} \sigma_{h}, \tau_{h}\right)_{h}:=\sum_{K \in \mathcal{T}_{h}}\left(\mathcal{A} \sigma_{h}, \tau_{h}\right)_{h, K}$ with

$$
\left(\mathcal{A} \sigma_{h}, \tau_{h}\right)_{h, K}:=I_{k, k^{\prime}, K}\left(\mathcal{A} \sigma_{h}: \tau_{h}\right),
$$

and $I_{k, k^{\prime}, K}$ is the quadrature operator in (3.2) satisfying the conditions (B1) and (B2). The following lemma shows that the quadrature rule (3.2) produces a coercive bilinear form $(\cdot, \cdot)_{h}$.

Lemma 4.1. It holds that

$$
(\mathcal{A} \tau, \tau)_{h} \gtrsim\|\tau\|_{0}^{2}, \quad \forall \tau \in \Sigma_{k, k^{\prime}, h}
$$


Proof. Recall that $\left\{\chi_{i}\right\}_{i=1}^{r}$ are the nodes for the $P_{k, k^{\prime}}$-Lagrange element, and $\left\{\zeta_{i}\right\}_{i=1}^{r}$ are the corresponding nodal basis functions satisfying (3.10). Then, for any $\tau \in \Sigma_{k, k^{\prime}, h}$ we can denote

$$
\left.\tau\right|_{K}=\sum_{i=1}^{r} \sum_{j=1}^{3} c_{i j} \zeta_{i} T_{i j},
$$

where $T_{i j} \in\left\{\mathbb{T}_{1}, \mathbb{T}_{2}, \mathbb{T}_{3}\right\}$ if $\chi_{i}$ is a vertex or a node inside $K$, and $T_{i j} \in\left\{T_{E}, T_{E, 1}^{\perp}, T_{E, 2}^{\perp}\right\}$ if $\chi_{i}$ is a node on edge $E$ (not the vertex). Thus,

$$
\begin{aligned}
(\mathcal{A} \tau, \tau)_{h, K} & =\left(\sum_{i=1}^{r}\left(\sum_{j=1}^{3} c_{i j} \mathcal{A} T_{i j}\right) \zeta_{i}, \sum_{s=1}^{r}\left(\sum_{t=1}^{3} c_{s t} T_{s t}\right) \zeta_{s}\right)_{h, K} \\
& =\sum_{i=1}^{r} \sum_{s=1}^{r} I_{k, k^{\prime}, K}\left(\left(\zeta_{i} \sum_{j=1}^{3} c_{i j} \mathcal{A} T_{i j}\right):\left(\zeta_{s} \sum_{t=1}^{3} c_{s t} T_{s t}\right)\right) \\
& =\sum_{i=1}^{r} w_{i}\left(\sum_{j=1}^{3} c_{i j} \mathcal{A} T_{i j}: \sum_{t=1}^{3} c_{i t} T_{i t}\right) \\
& \gtrsim \sum_{i=1}^{r} w_{i} \sum_{j=1}^{3} c_{i j}^{2} \gtrsim h^{2}\|\tau\|_{0, K}^{2},
\end{aligned}
$$

where $w_{i}$ are the weights in (3.2). As a result,

$$
(\mathcal{A} \tau, \tau)_{h} \gtrsim \sum_{K \in \mathcal{T}_{h}} h^{2}\|\tau\|_{0, K}^{2} \gtrsim\|\tau\|_{0}^{2},
$$

which completes the proof.

This coercivity lemma, together with the discrete BB condition (2.3), yields the following conclusion.

Lemma 4.2. The mass lumping scheme (4.1) admits a unique solution.

\subsection{Error estimation}

In light of the stability conditions (4.2) and (2.3) and standard techniques, we easily derive the following result.

Lemma 4.3. Let $(\sigma, u) \in \Sigma \times V$ and $\left(\sigma_{h}, u_{h}\right) \in \Sigma_{k, k^{\prime}, h} \times V_{k, k^{\prime}, h}$ be the solutions of (1.1) and (4.1), respectively. Then

$$
\begin{aligned}
& \left\|\sigma-\sigma_{h}\right\|_{H(\text { div })}+\left\|u-u_{h}\right\|_{0} \\
\lesssim & \left\|u-P_{h} u\right\|_{0}+\inf _{\tilde{\tau}_{h} \in \Sigma_{k, k^{\prime}, h}}\left(\left\|\sigma-\tilde{\tau}_{h}\right\|_{H(\text { div })}+\sup _{\tau_{h} \in \Sigma_{k, k^{\prime}, h}} \frac{E_{h}\left(\tilde{\tau}_{h}, \tau_{h}\right)}{\left\|\tau_{h}\right\|_{H(\operatorname{div})}}\right),
\end{aligned}
$$


where $P_{h}: L^{2}\left(\Omega ; \mathbb{R}^{2}\right) \mapsto V_{k, k^{\prime}, h}$ is the $L^{2}$ projection operator, and

$$
\begin{aligned}
E_{h}\left(\tilde{\tau}_{h}, \tau_{h}\right) & :=\left(\mathcal{A} \tilde{\tau}_{h}, \tau_{h}\right)-\left(\mathcal{A} \tilde{\tau}_{h}, \tau_{h}\right)_{h} \\
& =\sum_{K \in \mathcal{T}_{h}}\left(\int_{K} \mathcal{A} \tilde{\tau}_{h}: \tau_{h} d x-I_{k, k^{\prime}, K}\left(\mathcal{A} \tilde{\tau}_{h}: \tau_{h}\right)\right) .
\end{aligned}
$$

Let $W_{h}$ be a finite element space satisfying

$$
\Sigma_{k, h} \subseteq W_{h} \subseteq \Sigma_{k, k^{\prime}, h},
$$

and consisting of piecewise polynomial tensors of degree $\tilde{k}, k \leq \tilde{k} \leq k^{\prime}$. Then we have the following estimate for $E_{h}\left(\tilde{\tau}_{h}, \tau_{h}\right)$, which can be viewed as an extended version of [16, Lemma 5.2].

Lemma 4.4. If integers $p, q$ satisfy

$$
1 \leq p \leq k-1+\left(k^{\prime}-\tilde{k}\right), \quad 0 \leq q \leq 1,
$$

then for any $\left(\tilde{\tau}_{h}, \tau_{h}\right) \in \Sigma_{k, k^{\prime}, h} \times W_{h}$, it holds

$$
\left|E_{h}\left(\tilde{\tau}_{h}, \tau_{h}\right)\right| \lesssim h^{p+q}\left|\tau_{h}\right|_{p, h} \cdot\left|\tilde{\tau}_{h}\right|_{q, h} .
$$

Proof. For any $K=F_{K}(\hat{K}) \in \mathcal{T}_{h}$ with $x=F_{K}(\hat{x})$, we set

$$
\widehat{\tilde{\tau}_{h}}(\hat{x}):=\left.\tilde{\tau}_{h}(x)\right|_{K}, \quad \widehat{\tau_{h}}(\hat{x})=\left.\tau_{h}(x)\right|_{K}
$$

By scaling arguments we have

$$
\left|\widehat{\tilde{\tau}}_{h}\right|_{p, \hat{K}} \lesssim h^{p}\left|\operatorname{det} B_{K}\right|^{-\frac{1}{2}}\left|\tilde{\tau}_{h}\right|_{p, K}, \quad\left|\widehat{\tau}_{h}\right|_{p, \hat{K}} \lesssim h^{p}\left|\operatorname{det} B_{K}\right|^{-\frac{1}{2}}\left|\tau_{h}\right|_{p, K}
$$

Then

$$
\left|E_{h}\left(\tilde{\tau}_{h}, \tau_{h}\right)\right|=\sum_{K \in \mathcal{T}_{h}}\left|E_{h, K}\left(\tilde{\tau}_{h}, \tau_{h}\right)\right|=\sum_{K \in \mathcal{T}_{h}}\left|\operatorname{det} B_{K}\right| \hat{E}_{h, \hat{K}}\left(\widehat{\tilde{\tau}}_{h}, \widehat{\tau}_{h}\right) .
$$

From (4.5) it follows

$$
\begin{aligned}
& 0 \leq p-1+\tilde{k} \leq k+k^{\prime}-2 \\
& 0 \leq q-1+k^{\prime} \leq k+k^{\prime}-2 \\
& 0 \leq p-1+q-1 \leq k+k^{\prime}-2
\end{aligned}
$$

Let $\hat{\Pi}_{j}$ denote the $L^{2}$ projection from $\hat{L}^{2}(\hat{K} ; \mathbb{S})$ onto $\hat{P}_{j}(\hat{K} ; \mathbb{S})$. By (B2), the quadrature rule (3.2) is exact for $P_{k+k^{\prime}-2}$. In particular, we set $\hat{\Pi}_{-1}$ to be the zero operator. Thus,

$$
\begin{aligned}
&\left|\hat{E}_{h, \hat{K}}\left(\widehat{\tilde{\tau}_{h}}, \widehat{\tau_{h}}\right)\right|=\left|\hat{E}_{h, \hat{K}}\left(\widehat{\tilde{\tau}_{h}}-\hat{\Pi}_{p-1} \widehat{\tilde{\tau}_{h}}, \widehat{\tau_{h}}-\hat{\Pi}_{q-1} \widehat{\tau_{h}}\right)\right| \\
& \lesssim\left|\widehat{\widetilde{\tau}}_{h}-\hat{\Pi}_{p-1} \widehat{\tilde{\tau}_{h}}\right|_{0, \hat{K}} \cdot|| \widehat{\tau}_{h}-\left.\hat{\Pi}_{q-1} \widehat{\tau_{h}}\right|_{0, \hat{K}} \\
& \lesssim\left|\widehat{\tilde{\tau}_{h}}\right|_{p, \hat{K}} \cdot\left|\widehat{\tau}_{h}\right|_{q, \hat{K}} \lesssim h^{p}\left|\operatorname{det} B_{K}\right|^{-\frac{1}{2}}\left|\tilde{\tau}_{h}\right|_{p, K} \cdot h^{q}\left|\operatorname{det} B_{K}\right|^{-\frac{1}{2}}\left|\tau_{h}\right|_{q, K} \quad \text { by (4.7) } \\
& \lesssim h^{p+q}\left|\operatorname{det} B_{K}\right|^{-1}\left|\tilde{\tau}_{h}\right|_{p, K}\left|\tau_{h}\right|_{q, K},
\end{aligned}
$$

which,together with (4.8), yields the desired result. 
Remark 4.1. If taking $W_{h}=\Sigma_{k, k^{\prime}, h}$ and $p=k-1, q=0$ in Lemma 4.4, then we obtain

$$
\left|E_{h}\left(\tilde{\tau}_{h}, \tau_{h}\right)\right| \lesssim h^{k-1}\left|\tilde{\tau}_{h}\right|_{k-1, h} \cdot\left|\tau_{h}\right|_{0, h}, \quad \forall \tilde{\tau}_{h}, \tau_{h} \in \Sigma_{k, k^{\prime}, h},
$$

which yields

$$
\sup _{\tau_{h} \in \Sigma_{k, k^{\prime}, h}} \frac{E_{h}\left(\tilde{\tau}_{h}, \tau_{h}\right)}{\left\|\tau_{h}\right\|_{H(\mathrm{div})}} \lesssim h^{k-1}\left|\tilde{\tau}_{h}\right|_{k-1, h} .
$$

This inequality, together with Lemma 4.3, leads to an error estimate like

$$
\left\|\sigma-\sigma_{h}\right\|_{H(\operatorname{div})}+\left\|u-u_{h}\right\|_{0} \lesssim h^{k-1}\left(\|\sigma\|_{k}+\|u\|_{k-1}\right),
$$

provided that $\sigma \in H^{k}(\Omega, \mathbb{S})$ and $u \in H^{k-1}\left(\Omega, \mathbb{R}^{2}\right)$. Note that such an estimate is not optimal.

In what follows we will apply a more elaborate analysis to get a better estimate than (4.9) for the consistency error. To this end, we set, for any $K \in \mathcal{T}_{h}$,

$$
\Xi_{j}:=\underset{K \in \mathcal{T}_{h}}{\cup}\left(b P_{j}^{h o m}(K ; \mathbb{S})\right), \quad k-2 \leq j \leq k^{\prime}-3 .
$$

Here we recall that $P_{j}^{\text {hom }}(K ; \mathbb{S})$ denotes the set of homogeneous polynomial tensors of degree $j$. On the reference element $\hat{K}$ with vertexes $(0,0),(1,0)$ and $(0,1)$, the bubble function reads $\hat{b}=\hat{x}_{1} \hat{x}_{2}\left(1-\hat{x}_{1}-\hat{x}_{2}\right)$. Let $\left\{\hat{\psi}_{i}\right\}_{i=0}^{j}$ be the basis of the space $\hat{b} \hat{P}_{j}^{h o m}(\hat{K} ; \mathbb{R})$, then

$$
\hat{\psi}_{i}=\hat{x}_{1}^{i} \hat{x}_{2}^{j-i} \hat{b}=\hat{x}_{1}^{i+1} \hat{x}_{2}^{j-i+1}\left(1-\hat{x}_{1}-\hat{x}_{2}\right), \quad i=0,1, \ldots, j
$$

and

$$
\hat{\Xi}_{j}=\operatorname{span}\left\{\hat{\psi}_{i} \mathbb{T}_{s}: i=0,1, \ldots, j ; s=1,2,3\right\} .
$$

Lemma 4.5. It holds

$$
|\tau|_{1, h} \lesssim\|\operatorname{div} \tau\|_{0}, \quad \forall \tau \in \Xi_{j}, \quad j \geq 1
$$

Proof. By Lemma 3.2, it suffices to show that for $\forall \hat{\tau}=\sum_{i=0}^{j} \sum_{s=1}^{3} c_{i s} \hat{\psi}_{i} \mathbb{T}_{s} \in \hat{\Xi}_{j}$,

$$
\left.\widehat{\operatorname{div}} \hat{\tau}\right|_{\hat{K}}=\left(\begin{array}{c}
\sum_{i=0}^{j}\left(c_{i 1} \frac{\partial \hat{\psi}_{i}}{\partial \hat{x}_{1}}+c_{i 3} \frac{\partial \hat{\psi}_{i}}{\partial \hat{x}_{2}}\right) \\
\sum_{i=0}^{j}\left(c_{i 2} \frac{\partial \hat{\psi}_{i}}{\partial \hat{x}_{2}}+c_{i 3} \frac{\partial \hat{\psi}_{i}}{\partial \hat{x}_{1}}\right)
\end{array}\right)=0 \Rightarrow \hat{\tau}=0
$$

where $c_{i s}(i=0,1, \ldots, j ; s=1,2,3)$ are constants. To obtain (4.12) we only need to show that $\left\{\frac{\partial \hat{\psi}_{i}}{\partial \hat{x}_{1}}, \frac{\partial \hat{\psi}_{i}}{\partial \hat{x}_{2}}\right\}_{i=0}^{j}$ are linearly independent. In fact, we have

$$
\begin{aligned}
& \frac{\partial \hat{\psi}_{i}}{\partial \hat{x}_{1}}=(i+1) \hat{x}_{1}^{i} \hat{x}_{2}^{j-i+1}-(i+2) \hat{x}_{1}^{i+1} \hat{x}_{2}^{j-i+1}-(i+1) \hat{x}_{1}^{i} \hat{x}_{2}^{j-i+2}, \\
& \frac{\partial \hat{\psi}_{i}}{\partial \hat{x}_{2}}=(j-i+1) \hat{x}_{1}^{i+1} \hat{x}_{2}^{j-i}-(j-i+1) \hat{x}_{1}^{i+2} \hat{x}_{2}^{j-i}-(j-i+2) \hat{x}_{1}^{i+1} \hat{x}_{2}^{j-i+1} .
\end{aligned}
$$


Suppose that there are constants $\left\{c_{i}\right\}_{i=0}^{j}$ and $\left\{d_{i}\right\}_{i=0}^{j}$ such that

$$
\sum_{i=0}^{j} c_{i} \frac{\partial \hat{\psi}_{i}}{\partial \hat{x}_{1}}+\sum_{i=0}^{j} d_{i} \frac{\partial \hat{\psi}_{i}}{\partial \hat{x}_{2}}=0
$$

then for $0 \leq i \leq j+1$, it holds

$$
\begin{aligned}
& (i+1) c_{i}+(j-i+2) d_{i-1}=0 \\
& (i+1) c_{i}+(i+1) c_{i-1}+(j-i+3) d_{i-2}+(j-i+3) d_{i-1}=0
\end{aligned}
$$

where $c_{-1}=d_{-1}=d_{-2}=c_{j+1}=0$. Some simple calculations yield that

$$
c_{i}=d_{i}=0, \quad i=0,1, \ldots, j
$$

i.e. $\left\{\frac{\partial \hat{\psi}_{i}}{\partial \hat{x}_{1}}, \frac{\partial \hat{\psi}_{i}}{\partial \hat{x}_{2}}\right\}_{i=0}^{j}$ are linearly independent. This completes the proof.

Thanks to Lemma 4.5, we can obtain the following estimate for the consistency error.

Lemma 4.6. For any $\tilde{\tau}_{h} \in \Sigma_{k, k^{\prime}, h}$, it holds

$$
\sup _{\tau_{h} \in \Sigma_{k, k^{\prime}, h}} \frac{\left|E_{h}\left(\tilde{\tau}_{h}, \tau_{h}\right)\right|}{\left\|\tau_{h}\right\|_{H(\text { div })}} \lesssim h^{k}\left\|\tilde{\tau}_{h}\right\|_{k, h} .
$$

Proof. In view of the definition, (3.5), of $\Sigma_{k, k^{\prime}, h}$, for any $\tilde{\tau}_{h} \in \Sigma_{k, k^{\prime}, h}$ we have

$$
\sup _{\tau_{h} \in \Sigma_{k, k^{\prime}, h}} \frac{E_{h}\left(\tilde{\tau}_{h}, \tau_{h}\right)}{\left\|\tau_{h}\right\|_{H(\mathrm{div})}} \leq \sup _{\tau_{h} \in \Sigma_{k, k^{\prime}, h} \backslash \Xi_{k^{\prime}-3}} \frac{E_{h}\left(\tilde{\tau}_{h}, \tau_{h}\right)}{\left\|\tau_{h}\right\|_{H(\mathrm{div})}}+\sup _{\tau_{h} \in \Xi_{k^{\prime}-3}} \frac{E_{h}\left(\tilde{\tau}_{h}, \tau_{h}\right)}{\left\|\tau_{h}\right\|_{H(\mathrm{div})}}=: M_{1}+M_{2} .
$$

Since the degree of polynomials contained in $\Sigma_{k, k^{\prime}, h} \backslash \Xi_{k^{\prime}-3}$ is at most $k^{\prime}-1$, we can take $\tilde{k}=k^{\prime}-1, p=k, q=0$ in Lemma 4.4 to get

$$
M_{1}=\sup _{\tau_{h} \in \Sigma_{k, k^{\prime}, h} \backslash \Xi_{k^{\prime}-3}} \frac{E_{h}\left(\tilde{\tau}_{h}, \tau_{h}\right)}{\left\|\tau_{h}\right\|_{H(\operatorname{div})}} \lesssim h^{k}\left|\tilde{\tau}_{h}\right|_{k, h}
$$

To estimate $M_{2}$, we take $\tilde{k}=k^{\prime}, p=k-1, q=1$ in Lemma 4.4, then by Lemma 4.5 we obtain

$$
M_{2}=\sup _{\tau_{h} \in \Xi_{k^{\prime}-3}} \frac{E_{h}\left(\tilde{\tau}_{h}, \tau_{h}\right)}{\left\|\tau_{h}\right\|_{H(\operatorname{div})}} \lesssim \sup _{\tau_{h} \in \Xi_{k^{\prime}-3}} \frac{h^{k}\left|\tilde{\tau}_{h}\right|_{k-1, h}\left|\tau_{h}\right|_{1, h}}{\left\|\operatorname{div} \tau_{h}\right\|_{0}} \lesssim h^{k}\left|\tilde{\tau}_{h}\right|_{k-1, h}
$$

which, together with (4.14), yields the desired conclusion. 
Finally, combining Lemmas 4.6 and 4.3 immediately yields the following optimal error estimate for the mass lumping mixed finite element scheme.

Theorem 4.1. Let $(\sigma, u) \in\left(\Sigma \cap H^{k+1}(\Omega ; \mathbb{S})\right) \times\left(V \cap H^{k}\left(\Omega ; \mathbb{R}^{2}\right)\right)$ and $\left(\sigma_{h}, u_{h}\right) \in \Sigma_{k, k^{\prime}, h} \times$ $V_{k, k^{\prime}, h}$ solve (1.1) and (4.1), respectively. Then

$$
\left\|\sigma-\sigma_{h}\right\|_{H(\text { div })}+\left\|u-u_{h}\right\|_{0} \lesssim h^{k}\left(\|\sigma\|_{k+1}+\|u\|_{k}\right) .
$$

In what follows, we shall show that the use of finite element spaces $\Sigma_{k, k^{\prime}, h}-V_{k, k^{\prime}, h}$ with $k^{\prime}-k \geq 2$ in the scheme (4.1) leads to an optimal order accuracy for $\left\|\sigma-\sigma_{h}\right\|_{0}$.

Lemma 4.7. For any $\tau \in \Xi_{j} \oplus \Xi_{j+1}(j=1,2)$, if $\operatorname{div} \tau=0$, then $\tau=0$.

Proof. The proof is similar to that of (4.12). Let $\left\{\hat{\psi}_{i}\right\}_{i=0}^{j},\left\{\hat{\phi}_{i}\right\}_{i=0}^{j+1}$ be the basis of the space $\hat{b} \hat{P}_{j}^{h o m}(\hat{K} ; \mathbb{R})$ and $\hat{b} \hat{P}_{j+1}^{h o m}(\hat{K} ; \mathbb{R})$, respectively, where $\left\{\hat{\psi}_{i}\right\}_{i=0}^{j}$ are defined in (4.11) and

$$
\hat{\phi}_{i}=\hat{x}_{1}^{i} \hat{x}_{2}^{j-i+1} \hat{b}=\hat{x}_{1}^{i+1} \hat{x}_{2}^{j-i+2}\left(1-\hat{x}_{1}-\hat{x}_{2}\right), \quad i=0,1, \ldots, j+1 .
$$

Then it suffices to show that for

$$
\begin{aligned}
\hat{\tau} & =\sum_{s=1}^{3}\left(\sum_{i=0}^{j} c_{i s} \hat{\psi}_{i} \mathbb{T}_{s}+\sum_{i=0}^{j+1} d_{i s} \hat{\phi}_{i} \mathbb{T}_{s}\right) \in \widehat{\Xi}_{j} \oplus \widehat{\Xi}_{j+1}, \quad j=1,2, \\
\left.\widehat{\operatorname{div}} \hat{\tau}\right|_{\hat{K}} & =\left(\begin{array}{c}
\sum_{i=0}^{j}\left(c_{i 1} \frac{\partial \hat{\psi}_{i}}{\partial \hat{x}_{1}}+c_{i 3} \frac{\partial \hat{\psi}_{i}}{\partial \hat{x}_{2}}\right)+\sum_{i=0}^{j+1}\left(d_{i 1} \frac{\partial \hat{\phi}_{i}}{\partial \hat{x}_{1}}+d_{i 3} \frac{\partial \hat{\phi}_{i}}{\partial \hat{x}_{2}}\right) \\
\sum_{i=0}^{j}\left(c_{i 2} \frac{\partial \hat{\psi}_{i}}{\partial \hat{x}_{2}}+c_{i 3} \frac{\partial \hat{\psi}_{i}}{\partial \hat{x}_{1}}\right)+\sum_{i=0}^{j+1}\left(d_{i 2} \frac{\partial \hat{\phi}_{i}}{\partial \hat{x}_{2}}+d_{i 3} \frac{\partial \hat{\phi}_{i}}{\partial \hat{x}_{1}}\right)
\end{array}\right)=0
\end{aligned}
$$

implies $\hat{\tau}=0$, where $\left\{c_{i 1}, c_{i 2}, c_{i 3}\right\}_{i=0}^{j},\left\{d_{i 1}, d_{i 2}, d_{i 3}\right\}_{i=0}^{j+1}$ are constants. In fact, by comparing the coefficients of the term $\hat{x}_{1}^{i} \hat{x}_{2}^{j-i+3}(i=0,1, \ldots, j+3)$ in (4.16), we can get

$$
\begin{aligned}
& (i+1) d_{i 1}+(i+1) d_{i-1,1}+(j-i+4) d_{i-2,3}+(j-i+d) d_{i-1,3}=0 \\
& (i+1) d_{i 3}+(i+1) d_{i-1,3}+(j-i+4) d_{i-2,2}+(j-i+4) d_{i-1,2}=0
\end{aligned}
$$

where $d_{-2, s}=d_{-1, s}=d_{j+2, s}=d_{j+3, s}=0$ for $s=1,2,3$. Denote

$$
B_{1}:=\left[\begin{array}{ccccc}
1 & 0 & 0 & \cdots & 0 \\
2 & 2 & 0 & \cdots & 0 \\
0 & 3 & 3 & \cdots & 0 \\
0 & 0 & \ddots & \ddots & \vdots \\
0 & 0 & \cdots & j+2 & j+2 \\
\cdots & \cdots & \cdots & \cdots & j+3 \\
0 & 0 & 0 & \cdots & 0
\end{array}\right], \quad B_{2}:=\left[\begin{array}{ccccc}
0 & 0 & 0 & \cdots & 0 \\
j+3 & 0 & 0 & \cdots & 0 \\
j+2 & j+2 & 0 & \cdots & 0 \\
0 & j+1 & j+1 & \cdots & 0 \\
0 & 0 & \ddots & \ddots & \vdots \\
\cdots & \cdots & \cdots & 2 & 2 \\
0 & 0 & 0 & \cdots & 1
\end{array}\right],
$$

then the coefficient matrix of the linear system (4.17)-(4.18) can be denoted by

$$
B:=\left[\begin{array}{ccc}
B_{1} & O & B_{2} \\
O & B_{2} & B_{1}
\end{array}\right],
$$


and it is easy to derive that the rank of $B$ satisfies

$$
R(B)= \begin{cases}9, & j=1, \\ 12, & j=2 .\end{cases}
$$

Thus, $B$ is of column full rank. This means that $d_{i s}=0(i=0,1, \ldots, j+1 ; s=1,2,3)$. Notice that $\left\{\frac{\partial \hat{\psi}_{i}}{\partial \hat{x}_{1}}, \frac{\partial \hat{\psi}_{i}}{\partial \hat{x}_{2}}\right\}_{i=0}^{j}$ are linearly independent, which means that $c_{i s}=0(i=$ $0,1, \ldots, j ; s=1,2,3)$. As a result, we obtain $\hat{\tau}=0$.

Remark 4.2. For the case $j>2$, it is not easy to prove the conclusion of Lemma 4.7 in a unified way. However, one can check that case by case. For example, we have checked by using MATLAB that the conclusion also holds for $j \leq 40$.

Theorem 4.2. Under the conditions of Theorem 4.1 with $k^{\prime} \geq k+2$ and $k=3$, 4 , it holds

$$
\left\|\sigma-\sigma_{h}\right\|_{0} \lesssim h^{k+1}\|\sigma\|_{k+1}
$$

Proof. Let $\Pi_{h}$ be the same operator as in (3.23), then it suffices to show

$$
\left\|\Pi_{h} \sigma-\sigma_{h}\right\|_{0} \lesssim h^{k+1}\|\sigma\|_{k+1} .
$$

In fact, we can write

$$
\Pi_{h} \sigma-\sigma_{h}=\sigma_{1}+\sigma_{2}
$$

with $\sigma_{1} \in \Sigma_{k, k^{\prime}, h} \backslash\left(\Xi_{k^{\prime}-3} \oplus \Xi_{k^{\prime}-4}\right)$ and $\sigma_{2} \in \Xi_{k^{\prime}-3} \oplus \Xi_{k^{\prime}-4}$. By the community property (3.23), we get

$$
\operatorname{div}\left(\sigma_{1}+\sigma_{2}\right)=\operatorname{div}\left(\Pi_{h} \sigma-\sigma_{h}\right)=0 .
$$

Since the degrees of the polynomial tensors $\sigma_{1}$ and $\sigma_{2}$ are at most $k^{\prime}-2$ and $k^{\prime}$, respectively, the relation above means that the degree of $\operatorname{div} \sigma_{2}$ is at most $k^{\prime}-3$. Thus by Lemma 4.7, the degree of the polynomial tensor $\sigma_{2}$ is at most $k^{\prime}-2$. Thus the degree of that in $\Pi_{h} \sigma-\sigma_{h}$ is at most $k^{\prime}-2$.

Now we set $\tilde{k}=k^{\prime}-2, p=k+1, q=0$ in Lemma 4.4, then

$$
\begin{aligned}
& E_{h}\left(\Pi_{h} \sigma, \Pi_{h} \sigma-\sigma_{h}\right) \\
\lesssim & h^{k+1}\left|\Pi_{h} \sigma\right|_{k+1, h}\left\|\Pi_{h} \sigma-\sigma_{h}\right\|_{0} \lesssim h^{k+1}|\sigma|_{k+1}\left\|\Pi_{h} \sigma-\sigma_{h}\right\|_{0} .
\end{aligned}
$$

From (1.1), (4.1) and Lemma 4.1, it follows:

$$
\begin{aligned}
\left\|\Pi_{h} \sigma-\sigma_{h}\right\|_{0}^{2} & \lesssim\left(\mathcal{A}\left(\Pi_{h} \sigma-\sigma_{h}\right), \Pi_{h} \sigma-\sigma_{h}\right)_{h} \\
& =-\left(\mathcal{A}\left(\sigma-\Pi_{h} \sigma\right), \Pi_{h} \sigma-\sigma_{h}\right)-E_{h}\left(\Pi_{h} \sigma, \Pi_{h} \sigma-\sigma_{h}\right) .
\end{aligned}
$$

Combining the two estimates above indicates

$$
\left\|\Pi_{h} \sigma-\sigma_{h}\right\|_{0} \lesssim\left\|\sigma-\Pi_{h} \sigma\right\|_{0}+\frac{E_{h}\left(\Pi_{h} \sigma, \Pi_{h} \sigma-\sigma_{h}\right)}{\left\|\Pi_{h} \sigma-\sigma_{h}\right\|_{0}} \lesssim h^{k+1}\|\sigma\|_{k+1} .
$$

This finishes the proof. 


\section{Numerical results}

In this section, we shall give two numerical examples to verify our theoretical analysis for the scheme (2.1), of the modified mixed element $\Sigma_{k, k^{\prime}, h}-V_{k, k^{\prime}, h}$, and the mass lumping scheme (4.1) in three cases: $k=3, k^{\prime}=4 ; k=4, k^{\prime}=5 ; k=3, k^{\prime}=5$. In both examples, we take $\Omega=[0,1] \times[0,1]$ and use $N \times N$ uniform triangular meshes for the computation (cf. Fig. 2).

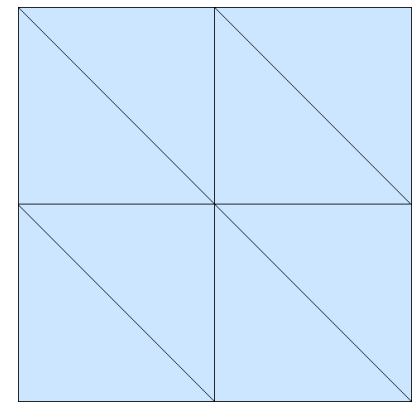

$2 \times 2$

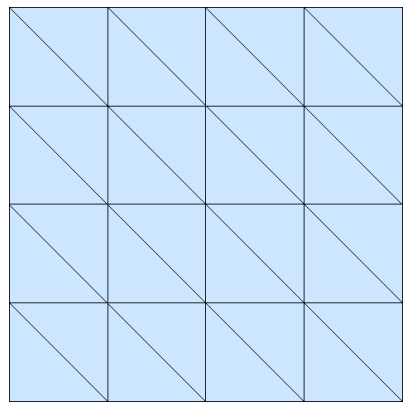

$4 \times 4$

Figure 2: The domain with uniform triangular meshes.

Example 5.1. Take Lamé constants $\mu=\frac{1}{2}, \lambda=1,10000$ in the model problem (1.1). $\Gamma_{D}=\partial \Omega$ and $\Gamma_{N}=\varnothing$. Let the exact solution $(\sigma, u)$ be of the following form:

$$
\begin{aligned}
u_{1}= & -x_{1}^{2} x_{2}\left(2 x_{2}-1\right)\left(x_{1}-1\right)^{2}\left(x_{2}-1\right), \\
u_{2}= & x_{1} x_{2}^{2}\left(2 x_{1}-1\right)\left(x_{2}-1\right)^{2}\left(x_{1}-1\right), \\
\sigma_{11}= & -\sigma_{22}=-2 x_{1} x_{2}\left(2 x_{1}^{2}-3 x_{1}+1\right)\left(2 x_{2}^{2}-3 x_{2}+1\right), \\
\sigma_{12}= & \sigma_{21}=x_{1} x_{2}^{2}\left(x_{2}-1\right)^{2}\left(2 x_{1}-\frac{3}{2}\right)-x_{1}^{2} x_{2}\left(x_{1}-1\right)^{2}\left(2 x_{2}-\frac{3}{2}\right) \\
& \quad-\frac{x_{1}^{2}}{2}\left(2 x_{2}-1\right)\left(x_{1}-1\right)^{2}\left(x_{2}-1\right)+\frac{x_{2}^{2}}{2}\left(2 x_{1}-1\right)\left(x_{1}-1\right)\left(x_{2}-1\right)^{2} .
\end{aligned}
$$

We list the error results of stress and displacement approximations in Tables 3-6. Table 3 shows the results of Hu-Zhang's element $\Sigma_{k, h}-V_{k, h}$ [25], the modified element $\Sigma_{k, k^{\prime}, h}-V_{k, k^{\prime}, h}$ and the mass lumping scheme for $k=3, k^{\prime}=4$. Table 4 demonstrates the results of the three methods for $k=4, k^{\prime}=5$. Table 5 gives the results of the modified element $\Sigma_{k, k^{\prime}, h}-V_{k, k^{\prime}, h}$ and the mass lumping scheme for $k=3, k^{\prime}=5$. We note that Tables 3-5 are all for $\lambda=1$. Table 6 lists the results with $\lambda=10000$ for the mass lumping scheme in three cases: $k=3, k^{\prime}=4 ; k=4, k^{\prime}=5 ; k=3, k^{\prime}=5$.

Example 5.2. We consider a case with mixed boundary conditions. Let $\Gamma_{D}=\{(x, y) \in$ $[0,1] \times[0,1], x=0$ or $x=1$ or $y=0\}, \Gamma_{N}=\{(x, y): x=0,0 \leq y \leq 1\}$. The boundary 
Table 3: History of convergence for Example 5.1: $\lambda=1, k=3, k^{\prime}=4$.

\begin{tabular}{||c|c|c|c|c|c|c|c||}
\hline \multirow{2}{*}{} & \multirow{2}{*}{$N$} & \multicolumn{2}{|c|}{$\frac{\left\|\sigma-\sigma_{h}\right\|_{0}}{\|\sigma\|_{0}}$} & \multicolumn{2}{|c||}{$\frac{\left\|\mathrm{div}\left(\sigma-\sigma_{h}\right)\right\|_{0}}{\| \text { div } \sigma \|_{0}}$} & \multicolumn{2}{c||}{$\frac{\left\|u-u_{h}\right\|_{0}}{\|u u\|_{0}}$} \\
\cline { 3 - 8 } Hu-Zhang's element & 2 & $9.361 \mathrm{e}-2$ & - & $9.256 \mathrm{e}-2$ & - & $1.409 \mathrm{e}-1$ & - \\
$\Sigma_{3, h}-V_{3, h}$ & 4 & $9.035 \mathrm{e}-3$ & 3.37 & $1.480 \mathrm{e}-2$ & 2.64 & $1.948 \mathrm{e}-2$ & 2.85 \\
& 8 & $6.498 \mathrm{e}-4$ & 3.79 & $1.953 \mathrm{e}-3$ & 2.92 & $2.590 \mathrm{e}-3$ & 2.91 \\
& 16 & $4.289 \mathrm{e}-5$ & 3.92 & $2.473 \mathrm{e}-4$ & 2.98 & $3.296 \mathrm{e}-4$ & 2.97 \\
& 32 & $2.742 \mathrm{e}-6$ & 3.96 & $3.102 \mathrm{e}-5$ & 2.99 & $4.139 \mathrm{e}-5$ & 2.99 \\
\hline \multirow{3}{*}{ Modified element } & 2 & $1.065 \mathrm{e}-1$ & - & $5.414 \mathrm{e}-2$ & - & $7.038 \mathrm{e}-2$ & - \\
$\Sigma_{3,4, h}-V_{3,4, h}$ & 4 & $1.120 \mathrm{e}-2$ & 3.25 & $7.438 \mathrm{e}-3$ & 2.86 & $9.685 \mathrm{e}-3$ & 2.86 \\
& 8 & $8.296 \mathrm{e}-4$ & 3.75 & $9.496 \mathrm{e}-4$ & 2.96 & $1.240 \mathrm{e}-3$ & 2.96 \\
& 16 & $5.551 \mathrm{e}-5$ & 3.90 & $1.193 \mathrm{e}-4$ & 2.99 & $1.565 \mathrm{e}-4$ & 2.98 \\
& 32 & $3.573 \mathrm{e}-6$ & 3.95 & $1.493 \mathrm{e}-5$ & 3.00 & $1.962 \mathrm{e}-5$ & 2.99 \\
\hline \multirow{3}{*}{ Mass lumping } & 2 & $1.219 \mathrm{e}-1$ & - & $6.417 \mathrm{e}-2$ & - & $8.983 \mathrm{e}-2$ & - \\
$\Sigma_{3,4, h}-V_{3,4, h}$ & 4 & $1.731 \mathrm{e}-2$ & 2.81 & $7.880 \mathrm{e}-3$ & 3.02 & $1.327 \mathrm{e}-2$ & 2.75 \\
& 8 & $2.0759 \mathrm{e}-3$ & 3.06 & $9.741 \mathrm{e}-4$ & 3.01 & $1.758 \mathrm{e}-3$ & 2.91 \\
& 16 & $2.466 \mathrm{e}-4$ & 3.07 & $1.213 \mathrm{e}-4$ & 3.00 & $2.232 \mathrm{e}-4$ & 2.97 \\
& 32 & $2.981 \mathrm{e}-5$ & 3.04 & $1.515 \mathrm{e}-5$ & 3.00 & $2.801 \mathrm{e}-5$ & 2.99 \\
\hline
\end{tabular}

Table 4: History of convergence for Example 5.1: $\lambda=1, k=4, k^{\prime}=5$.

\begin{tabular}{|c|c|c|c|c|c|c|c|}
\hline & \multirow[t]{2}{*}{$N$} & \multicolumn{2}{|c|}{$\frac{\left\|\sigma-\sigma_{h}\right\|_{0}}{\|\sigma\|_{0}}$} & \multicolumn{2}{|c|}{$\frac{\left\|\operatorname{div}\left(\sigma-\sigma_{h}\right)\right\|_{0}}{\|\operatorname{div} \sigma\|_{0}}$} & \multicolumn{2}{|c|}{$\frac{\left\|u-u_{h}\right\|_{0}}{\|u\|_{0}}$} \\
\hline & & Error & Order & Error & Order & Error & Order \\
\hline \multirow{5}{*}{$\begin{array}{l}\text { Hu-Zhang's element } \\
\qquad \Sigma_{4, h}-V_{4, h}\end{array}$} & 2 & $1.919 \mathrm{e}-2$ & - & $2.505 \mathrm{e}-2$ & - & $2.583 \mathrm{e}-2$ & - \\
\hline & 4 & $7.329 \mathrm{e}-4$ & 4.71 & $1.724 \mathrm{e}-3$ & 3.86 & $2.655 e-3$ & 3.28 \\
\hline & 8 & $2.481 e-5$ & 4.88 & $1.101 \mathrm{e}-4$ & 3.96 & $1.860 \mathrm{e}-4$ & 3.83 \\
\hline & 16 & $8.043 e-7$ & 4.94 & $6.919 \mathrm{e}-6$ & 3.99 & $1.194 \mathrm{e}-5$ & 3.96 \\
\hline & 32 & $2.557 \mathrm{e}-8$ & 4.97 & $4.330 \mathrm{e}-7$ & 4.00 & $7.519 \mathrm{e}-7$ & 3.99 \\
\hline \multirow{5}{*}{$\begin{array}{c}\text { Modified element } \\
\Sigma_{4,5, h}-V_{4,5, h}\end{array}$} & 2 & $2.602 \mathrm{e}-2$ & - & $4.862 \mathrm{e}-3$ & - & $1.403 \mathrm{e}-2$ & - \\
\hline & 4 & $9.792 \mathrm{e}-4$ & 4.73 & $2.239 \mathrm{e}-4$ & 4.44 & $6.087 \mathrm{e}-4$ & 4.52 \\
\hline & 8 & $3.302 \mathrm{e}-5$ & 4.88 & $1.243 e-5$ & 4.17 & $3.298 \mathrm{e}-5$ & 4.20 \\
\hline & 16 & $1.069 \mathrm{e}-6$ & 4.94 & $7.508 \mathrm{e}-7$ & 4.04 & $1.980 \mathrm{e}-6$ & 4.05 \\
\hline & 32 & $3.401 \mathrm{e}-8$ & 4.97 & $4.650 \mathrm{e}-8$ & 4.01 & $1.225 \mathrm{e}-7$ & 4.01 \\
\hline \multirow{5}{*}{$\begin{array}{l}\text { Mass lumping } \\
\Sigma_{4,5, h}-V_{4,5, h}\end{array}$} & 2 & $3.679 \mathrm{e}-2$ & - & $6.097 e-3$ & - & $1.751 \mathrm{e}-2$ & - \\
\hline & 4 & $2.377 \mathrm{e}-3$ & 3.95 & $2.532 \mathrm{e}-4$ & 4.58 & $1.753 \mathrm{e}-3$ & 3.32 \\
\hline & 8 & $1.499 \mathrm{e}-4$ & 3.98 & $1.308 \mathrm{e}-5$ & 4.27 & $1.223 \mathrm{e}-4$ & 3.84 \\
\hline & 16 & $9.369 \mathrm{e}-6$ & 4.00 & $7.690 \mathrm{e}-7$ & 4.08 & $7.853 e-6$ & 3.96 \\
\hline & 32 & $5.843 e-7$ & 4.00 & $4.727 e-8$ & 4.02 & $4.942 \mathrm{e}-7$ & 3.99 \\
\hline
\end{tabular}


Table 5: History of convergence for Example 5.1: $\lambda=1, k=3, k^{\prime}=5$.

\begin{tabular}{||c|c|c|c|c|c|c|c||}
\hline & \multirow{2}{*}{$N$} & \multicolumn{2}{|c|}{$\frac{\left\|\sigma-\sigma_{h}\right\|_{0}}{\|\sigma\|_{0}}$} & \multicolumn{2}{c|}{$\frac{\left\|\operatorname{div}\left(\sigma-\sigma_{h}\right)\right\|_{0}}{\|\operatorname{div} \sigma\|_{0}}$} & \multicolumn{2}{c||}{$\frac{\left\|u-u_{h}\right\|_{0}}{\|u\|_{0}}$} \\
\cline { 3 - 8 } & & Error & Order & Error & Order & Error & Order \\
\hline \multirow{3}{*}{ Modified element } & 2 & $1.140 \mathrm{e}-1$ & - & $3.226 \mathrm{e}-2$ & - & $5.201 \mathrm{e}-2$ & - \\
$\Sigma_{3,5, h}-V_{3,5, h}$ & 4 & $1.185 \mathrm{e}-2$ & 3.26 & $4.176 \mathrm{e}-3$ & 2.95 & $5.757 \mathrm{e}-3$ & 3.17 \\
& 8 & $8.745 \mathrm{e}-4$ & 3.76 & $5.248 \mathrm{e}-4$ & 2.99 & $6.694 \mathrm{e}-4$ & 3.10 \\
& 16 & $5.841 \mathrm{e}-5$ & 3.90 & $6.567 \mathrm{e}-5$ & 3.00 & $8.354 \mathrm{e}-5$ & 3.00 \\
& 32 & $3.757 \mathrm{e}-6$ & 3.95 & $8.211 \mathrm{e}-6$ & 3.00 & $1.045 \mathrm{e}-5$ & 3.00 \\
\hline \multirow{3}{*}{ Mass lumping } & 2 & $1.131 \mathrm{e}-1$ & - & $3.575 \mathrm{e}-2$ & - & $6.825 \mathrm{e}-2$ & - \\
$\Sigma_{3,5, h}-V_{3,5, h}$ & 4 & $1.184 \mathrm{e}-2$ & 3.25 & $4.621 \mathrm{e}-3$ & 2.95 & $6.751 \mathrm{e}-3$ & 3.33 \\
& 8 & $8.751 \mathrm{e}-4$ & 3.75 & $5.809 \mathrm{e}-4$ & 2.99 & $7.929 \mathrm{e}-4$ & 3.08 \\
& 16 & $5.850 \mathrm{e}-5$ & 3.90 & $7.271 \mathrm{e}-5$ & 3.00 & $9.862 \mathrm{e}-5$ & 3.00 \\
& 32 & $3.764 \mathrm{e}-6$ & 3.95 & $9.091 \mathrm{e}-6$ & 3.00 & $1.233 \mathrm{e}-5$ & 3.00 \\
\hline
\end{tabular}

Table 6: History of convergence for Example 5.1: $\lambda=10000$.

\begin{tabular}{|c|c|c|c|c|c|c|c|}
\hline & \multirow{2}{*}{$N$} & \multicolumn{2}{|c|}{$\frac{\left\|\sigma-\sigma_{h}\right\|_{0}}{\|\sigma\|_{0}}$} & \multicolumn{2}{|c|}{$\frac{\left\|\operatorname{div}\left(\sigma-\sigma_{h}\right)\right\|_{0}}{\|\operatorname{div} \sigma\|_{0}}$} & \multicolumn{2}{|c|}{$\frac{\left\|u-u_{h}\right\|_{0}}{\|u\|_{0}}$} \\
\hline & & Error & Order & Error & Order & Error & Order \\
\hline \multirow{5}{*}{$\begin{array}{l}\text { Mass lumping } \\
\Sigma_{3,4, h}-V_{3,4, h}\end{array}$} & 2 & $1.286 \mathrm{e}-1$ & - & $6.418 \mathrm{e}-2$ & - & $9.141 \mathrm{e}-2$ & - \\
\hline & 4 & $1.974 \mathrm{e}-2$ & 2.70 & $7.881 \mathrm{e}-3$ & 3.03 & $1.338 \mathrm{e}-2$ & 2.77 \\
\hline & 8 & $2.516 \mathrm{e}-3$ & 2.79 & $9.741 \mathrm{e}-4$ & 3.02 & $1.764 \mathrm{e}-3$ & 2.92 \\
\hline & 16 & $3.082 \mathrm{e}-4$ & 3.03 & $1.214 \mathrm{e}-4$ & 3.00 & $2.235 \mathrm{e}-4$ & 2.98 \\
\hline & 32 & $3.7824 \mathrm{e}-5$ & 3.03 & $1.516 \mathrm{e}-5$ & 3.00 & $2.803 e-5$ & 3.00 \\
\hline \multirow{5}{*}{$\begin{array}{l}\text { Mass lumping } \\
\Sigma_{4,5, h}-V_{4,5, h}\end{array}$} & 2 & $4.186 \mathrm{e}-2$ & - & $6.098 \mathrm{e}-3$ & - & $1.787 \mathrm{e}-2$ & - \\
\hline & 4 & $2.910 \mathrm{e}-3$ & 3.85 & $2.532 \mathrm{e}-4$ & 4.59 & $1.753 e-3$ & 3.35 \\
\hline & 8 & $1.880 \mathrm{e}-4$ & 3.95 & $1.309 \mathrm{e}-5$ & 4.27 & $1.223 \mathrm{e}-4$ & 3.84 \\
\hline & 16 & $1.182 \mathrm{e}-5$ & 3.99 & 7.691e-7 & 4.09 & $7.853 e-6$ & 3.96 \\
\hline & 32 & $7.375 \mathrm{e}-7$ & 4.00 & $4.727 e-8$ & 4.02 & $4.942 \mathrm{e}-7$ & 3.99 \\
\hline \multirow{5}{*}{$\begin{array}{l}\text { Mass lumping } \\
\Sigma_{3,5, h}-V_{3,5, h}\end{array}$} & 2 & $1.37 \mathrm{e}-1$ & - & $3.575 \mathrm{e}-2$ & - & $5.737 e-2$ & - \\
\hline & 4 & $1.194 \mathrm{e}-2$ & 3.25 & $4.622 \mathrm{e}-3$ & 2.95 & $6.489 e-3$ & 3.14 \\
\hline & 8 & $8.809 e-4$ & 3.76 & $5.810 \mathrm{e}-4$ & 2.99 & $7.906 \mathrm{e}-4$ & 3.04 \\
\hline & 16 & $5.874 \mathrm{e}-5$ & 3.91 & $7.271 \mathrm{e}-5$ & 3.00 & $9.865 e-5$ & 3.00 \\
\hline & 32 & $3.773 e-6$ & 3.96 & $9.092 \mathrm{e}-6$ & 3.00 & $1.2335 \mathrm{e}-5$ & 3.00 \\
\hline
\end{tabular}

conditions are $u=g$ on $\Gamma_{D}$ and $\sigma n=\kappa$ on $\Gamma_{N}$. We take $\lambda=1, \mu=\frac{1}{2}$. Let the exact solution $(\sigma, u)$ be of the following form:

$$
\begin{aligned}
& u_{1}=\left(1-\mu^{2}\right) \sin \left(\pi x_{1}\right) \sin \left(\pi x_{2}\right), \\
& u_{2}=\mu \sin \left(\pi x_{1}\right) \sin \left(\pi x_{2}\right), \\
& \sigma_{11}=\pi \lambda \mu \cos \left(\pi x_{2}\right) \sin \left(\pi x_{1}\right)-\pi\left(\mu^{2}-1\right)(\lambda+2 \mu) \cos \left(\pi x_{1}\right) \sin \left(\pi x_{2}\right), \\
& \sigma_{22}=\pi \mu(\lambda+2 \mu) \cos \left(\pi x_{2}\right) \sin \left(\pi x_{1}\right)-\pi \lambda\left(\mu^{2}-1\right) \cos \left(\pi x_{1}\right) \sin \left(\pi x_{2}\right),
\end{aligned}
$$




$$
\sigma_{12}=\sigma_{21}=-\mu \pi\left(\mu^{2}-1\right) \cos \left(\pi x_{2}\right) \sin \left(\pi x_{1}\right)+\pi \mu^{2} \cos \left(\pi x_{1}\right) \sin \left(\pi x_{2}\right) .
$$

Table 7 shows the results for the mass lumping for Example 5.2 in three cases: $k=$ $3, k^{\prime}=4 ; k=4, k^{\prime}=5 ; k=3, k^{\prime}=5$.

From the numerical results in Tables 3-7, we have the following observations:

- As same as Hu-Zhang's element, the modified element $\Sigma_{k, k^{\prime}, h}-V_{k, k^{\prime}, h}$ for $k=3,4$ yields the $k$-th order of convergence for $\left\|\operatorname{div}\left(\sigma-\sigma_{h}\right)\right\|_{0}$ and $\left\|u-u_{h}\right\|_{0}$, and $k+1$-th order of convergence for $\left\|\sigma-\sigma_{h}\right\|_{0}$. This is conformable to the theoretical results in Theorem 3.2.

- The mass lumping scheme of the modified element $\Sigma_{k, k^{\prime}, h}-V_{k, k^{\prime}, h}$ yields the $k$-th order of convergence for $\left\|\operatorname{div}\left(\sigma-\sigma_{h}\right)\right\|_{0}$ and $\left\|u-u_{h}\right\|_{0}$, as is conformable to the theoretical result in Theorem 4.1.

- The mass lumping scheme of $\Sigma_{k, k^{\prime}, h}-V_{k, k^{\prime}, h}$, with $k=3, k^{\prime}=4$ and $k=4, k^{\prime}=5$, yields the $k$-th order of convergence for $\left\|\sigma-\sigma_{h}\right\|_{0}$, one order lower than the original scheme, while the mass lumping scheme with $k=3, k^{\prime}=5$ yields the $k+1$-th order of convergence, which is consistent with Theorem 4.2.

- Though the proposed modified element $\Sigma_{k, k^{\prime}, h}-V_{k, k^{\prime}, h}$ is of more degrees of freedom than the original Hu-Zhang's element $\Sigma_{k, h}-V_{k, h}$, its mass lumping scheme leads to a SPD system that is much easier to solve.

- The mass lumping schemes are robust with respect to the Lamé constant $\lambda$.

Table 7: History of convergence for Example 5.2.

\begin{tabular}{|c|c|c|c|c|c|c|c|}
\hline & \multirow{2}{*}{$N$} & \multicolumn{2}{|c|}{$\frac{\left\|\sigma-\sigma_{h}\right\|_{0}}{\|\sigma\|_{0}}$} & \multicolumn{2}{|c|}{$\frac{\left\|\operatorname{div}\left(\sigma-\sigma_{h}\right)\right\|_{0}}{\|\operatorname{div} \sigma\|_{0}}$} & \multicolumn{2}{|c|}{$\frac{\left\|u-u_{h}\right\|_{0}}{\|u\|_{0}}$} \\
\hline & & Error & Order & Error & Order & Error & Order \\
\hline \multirow{5}{*}{$\begin{array}{l}\text { Mass lumping } \\
\Sigma_{3,4, h}-V_{3,4, h}\end{array}$} & 2 & $2.139 \mathrm{e}-2$ & - & $2.600 \mathrm{e}-2$ & - & $3.129 \mathrm{e}-2$ & - \\
\hline & 4 & $1.982 \mathrm{e}-3$ & 3.43 & $3.184 \mathrm{e}-3$ & 3.02 & $4.239 \mathrm{e}-3$ & 2.88 \\
\hline & 8 & $1.879 \mathrm{e}-4$ & 3.39 & $3.949 e-4$ & 3.01 & $5.547 e-4$ & 2.93 \\
\hline & 16 & $2.098 \mathrm{e}-5$ & 3.16 & $4.926 \mathrm{e}-5$ & 3.00 & $7.034 \mathrm{e}-5$ & 2.97 \\
\hline & 32 & $2.699 \mathrm{e}-6$ & 2.95 & $6.154 \mathrm{e}-6$ & 3.00 & $8.826 \mathrm{e}-6$ & 2.99 \\
\hline \multirow{5}{*}{$\begin{array}{l}\text { Mass lumping } \\
\Sigma_{4,5, h}-V_{4,5, h}\end{array}$} & 2 & $3.624 \mathrm{e}-3$ & - & $3.331 \mathrm{e}-3$ & - & $4.372 \mathrm{e}-3$ & - \\
\hline & 4 & $1.607 \mathrm{e}-4$ & 4.49 & $1.985 \mathrm{e}-4$ & 4.06 & $2.878 \mathrm{e}-4$ & 3.92 \\
\hline & 8 & $7.763 e-6$ & 4.37 & $1.221 \mathrm{e}-5$ & 4.02 & $1.880 \mathrm{e}-5$ & 3.93 \\
\hline & 16 & $4.319 \mathrm{e}-7$ & 4.16 & 7.601e-7 & 4.00 & $1.192 \mathrm{e}-6$ & 3.97 \\
\hline & 32 & $2.605 e-8$ & 4.05 & $4.745 e-8$ & 4.00 & $7.480 \mathrm{e}-8$ & 3.99 \\
\hline \multirow{5}{*}{$\begin{array}{l}\text { Mass lumping } \\
\Sigma_{3,5, h}-V_{3,5, h}\end{array}$} & 2 & $1.921 \mathrm{e}-2$ & - & $1.064 \mathrm{e}-2$ & - & $3.262 \mathrm{e}-2$ & - \\
\hline & 4 & $1.601 \mathrm{e}-3$ & 3.58 & $1.389 \mathrm{e}-3$ & 2.93 & $1.985 \mathrm{e}-3$ & 4.03 \\
\hline & 8 & $1.075 \mathrm{e}-4$ & 3.89 & $1.761 \mathrm{e}-4$ & 2.98 & $1.793 \mathrm{e}-4$ & 3.46 \\
\hline & 16 & $6.858 \mathrm{e}-6$ & 3.97 & $2.209 \mathrm{e}-5$ & 2.99 & $2.111 \mathrm{e}-5$ & 3.08 \\
\hline & 32 & $4.318 \mathrm{e}-7$ & 3.98 & $2.765 e-6$ & 2.99 & $2.610 \mathrm{e}-6$ & 3.01 \\
\hline
\end{tabular}




\section{Acknowledgments}

This work was supported in part by the National Natural Science Foundation of China (Grants 11771312, 12171340).

\section{References}

[1] S. AdAMS AND B. COCKBURn, A mixed finite element method for elasticity in three dimensions, J. Sci. Comput. 25 (2005), 515-521.

[2] D. N. ARnold AND G. Awanou, Rectangular mixed finite elements for elasticity, Math. Models Methods Appl. Sci. 15 (2005), 1417-1429.

[3] D. N. ARnold, G. Awanou, And R. Winther, Finite elements for symmetric tensors in three dimensions, Math. Comp. 77 (2008), 1229-1251.

[4] D. N. ARNold, J. D. JR, AND C. P. GuPTA, A family of higher order mixed finite element methods for plane elasticity, Numer. Math. 45 (1984), 1-22.

[5] D. N. ARnold AND R. Winther, Mixed finite elements for elasticity, Numer. Math. 92 (2002), 401-419.

[6] D. N. ARnold And R. Winther, Nonconforming mixed elements for elasticity, Math. Models Methods Appl. Sci. 13 (2003), 295-307.

[7] G. AwANOU, Two remarks on rectangular mixed finite elements for elasticity, J. Sci. Comput. 50 (2012), 91-102.

[8] G. A. BAKER AND V. A. Dougalis, The effect of quadrature errors on finite element approximations for second order hyperbolic equations, SIAM J. Numer. Anal. 13 (1976), 577-598.

[9] E. BÉCACHE, P. JolY, AND C. TSOGKA, A new family of mixed finite elements for the linear elastodynamic problem, SIAM J. Numer. Anal. 39 (2002), 2109-2132.

[10] F. BREZZI, On the existence, uniqueness and approximation of saddle-point problems arising from Lagrangian multipliers, R.A.I.R.O Analyse Numérique 8 (1974), 129-151.

[11] F. BRezZi AND M. Fortin, Mixed and hybrid finite element methods, Springer-Verlag, 1991.

[12] S. C. Chen AND Y. N. WANG, Conforming rectangular mixed finite elements for elasticity, J. Sci. Comput. 47 (2011), 93-108.

[13] M. J. S. Chin-Joe-Kong, W. A. Mulder, And M. V. Veldhuizen, Higher-order triangular and tetrahedral finite elements with mass lumping for solving the wave equation, J. Engrg. Math. 35 (1999), 405-426.

[14] P. G. Ciarlet, The finite element method for elliptic problems, North-Holland Pub. Co, 1978.

[15] G. Cohen And S. Fauqueux, Mixed spectral finite elements for the linear elasticity system in unbounded domains, SIAM J. Sci. Comput. 26(3) (2005), 864-884.

[16] G. COHEN, P. JOLY, J. E. ROBERTS, AND N. TORDJMAN, Higher order triangular finite elements with mass lumping for the wave equation, SIAM J. Numer. Anal. 38 (2001), 20472078.

[17] T. CUI, W. LENG, D. Lin, S. MA, AND L. ZHANG, High order mass-lumping finite elements on simplexes, Numer. Math. Theor. Meth. Appl. 10(2) (2017), 331-350.

[18] G. J. FIX, Effects of quadrature errors in finite element approximation of steady state, eigenvalue and parabolic problems, in: The Mathematical Foundations of the Finite Element Method with Applications to Partial Differential Equations, (1972), 525-556. 
[19] F. X. Giraldo AND M. A. TAYlor, A diagonal-mass-matrix triangular-spectral element method based on cubature points, J. Engrg. Math. 56 (2006), 307-322.

[20] J. GOPALAKRISHNAN AND J. GUZMÁN, Symmetric nonconforming mixed finite elements for linear elasticity, SIAM J. Numer. Anal. 49 (2011), 1504-1520.

[21] J. Hu, Finite element approximations of symmetric tensors on simplicial grids in $R^{n}$ : the high order case, J. Comput. Math. 33 (2015), 283-296.

[22] J. HU AND R. MA, Nonconforming mixed finite elements for linear elasticity on simplicial grids, Numer. Methods Partial Differential Equations (2018), 1-17.

[23] J. Hu, H. Y. MAN, AND S. Y. ZHANG, A simple conforming mixed finite element for linear elasticity on rectangular grids in any space dimension, J. Sci. Comput. 58 (2014), 367-379.

[24] J. HU AND Z. C. SHI, Lower order rectangular nonconforming mixed finite elements for plane elasticity, SIAM J. Numer. Anal. 46 (2007), 88-102.

[25] J. HU AND S. Y. ZHANG, A family of conforming mixed finite elements for linear elasticity on triangular grids, arXiv:1406.7457, (2014).

[26] J. HU AND S. Y. ZHANG, A family of symmetric mixed finite elements for linear elasticity on tetrahedral grids, Science China Mathematics 58 (2015), 297-307.

[27] Y. S. LIU, J. W. TENG, T. XU, AND J. BADAL, Higher-order triangular spectral element method with optimized cubature points for seismic wavefield modeling, J. Comput. Phys. 336 (2017), 458-480.

[28] W. A. MULDER, Higher-order mass-lumped finite elements for the wave equation, Journal of Computational Acoustics 9 (2001), 671-680.

[29] W. A. Mulder, New triangular mass-lumped finite elements of degree 6 for wave propagation, Progress in Electromagnetics Research 141 (2013), 671-692.

[30] P. A. RAVIART, The use of numerical integration in finite element methods for solving parabolic equations, Topics in numerical analysis (Proc. Roy. Irish Acad. Conf., University Coll., Dublin, 1972), Academic Press, (1973), 233-264.

[31] X. XIE AND J. XU, New mixed finite elements for plane elasticity and Stokes equation, Science China Mathematics 54 (2011), 1499-1519.

[32] S. Y. YI, A new nonconforming mixed finite element method for linear elasticity, Math. Models Methods Appl. Sci. 16 (2006), 979-999.

[33] A. Younes, P. ACKerer, AND F. Lehmann, A new mass lumping scheme for the mixed hybrid finite element method, Internat. J. Numer. Methods Engrg. 67 (2006), 89-107. 